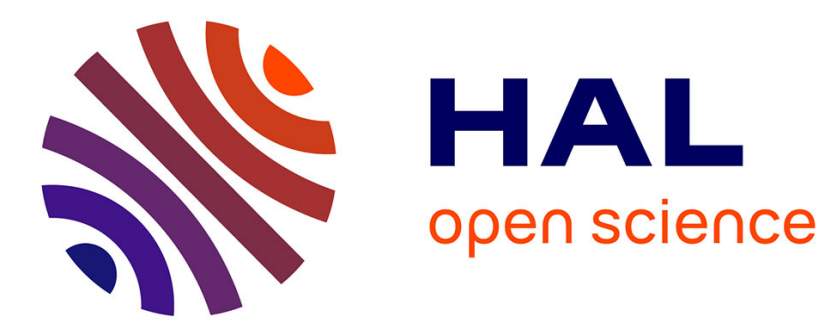

\title{
Interpréter les signes du dieu : une apparition de Mandoulis au temple de Kalabchah
}

Gaëlle Tallet

\section{To cite this version:}

Gaëlle Tallet. Interpréter les signes du dieu: une apparition de Mandoulis au temple de Kalabchah. Georgoudi Stella; Koch-Piettre Renée; Schmidt Francis. La Raison des signes. Présages, rites, destins dans les sociétés de la Méditerranée ancienne, 174, Brill, pp.343-383, 2012, Religions in the GraecoRoman World. hal-01374244

\section{HAL Id: hal-01374244 https://hal.science/hal-01374244}

Submitted on 17 Nov 2016

HAL is a multi-disciplinary open access archive for the deposit and dissemination of scientific research documents, whether they are published or not. The documents may come from teaching and research institutions in France or abroad, or from public or private research centers.
L'archive ouverte pluridisciplinaire HAL, est destinée au dépôt et à la diffusion de documents scientifiques de niveau recherche, publiés ou non, émanant des établissements d'enseignement et de recherche français ou étrangers, des laboratoires publics ou privés. 


\title{
La Raison des signes
}

\section{Présages, rites, destin dans les sociétés de la Méditerranée ancienne}

\author{
Sous la direction de \\ Stella Georgoudi \\ Renée Koch Piettre \\ Francis Schmidt
}

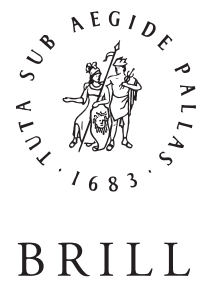

LEIDEN • BOSTON

2012

(C) 2012 Koninklijke Brill NV 
TABLE DES MATIÈRES

Remerciements …................................................................... ix

Table des illustrations ………….................................................... xi

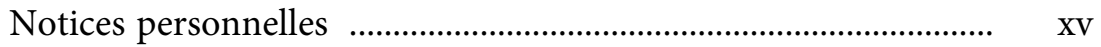

Présentation .........................................................................................

Stella Georgoudi, Renée Koch Piettre et Francis Schmidt

PREMIÈRE PARTIE

INSTITUTIONS DIVINATOIRES ET CONSTRUCTION RITUELLE DES SIGNES

La fabrique des présages en Mésopotamie: la sémiologie des devins

Jean-Jacques Glassner

Des sons, des signes et des paroles: la divination à l'œuvre dans l'oracle de Dodone Stella Georgoudi

Hermès et la mantique grecque 91 Dominique Jaillard

Le rite des auspices à Rome: quelle évolution? Réflexions sur la transformation de la divination publique des Romains entre le $\mathrm{III}^{\mathrm{e}}$ et le $\mathrm{I}^{\text {er }}$ siècle avant notre ère 109 John Scheid

DEUXIÈME PARTIE

SIGNES IMPROMPTUS ET PHÉNOMÈNES NATURELS. PRÉSAGES ET PRODIGES

Les signes de la nature dans l'Égypte pharaonique 131 Emmanuel Jambon 
Le corbeau: un signe dans le monde grec

Atome ou Providence? La Vie de Timoléon de Plutarque, ou comment faire de l'Histoire avec des atomes

Renée Koch Piettre

Teras ou les modalités du prodige dans le discours divinatoire grec: une perspective comparatiste

Ileana Chirassi Colombo

Signes et prodiges chez Flavius Josèphe et Tacite

(Guerre des Juifs VI, 288-315; Histoires V, 13)

Francis Schmidt

TROISIĖME PARTIE

SIGNES DE L'INTERVENTION DIVINE:

DE L'ÉLECTION À LA LÉGITIMATION

Les signes divins au service du pouvoir sacerdotal en Anatolie hellénistique et romaine Laetitia Bernadet

«Un châtiment en adviendra». Le malheur comme signe des dieux dans l'Anatolie impériale Nicole Belayche

Interpréter les signes du dieu: une apparition de Mandoulis au temple de Kalabchah

Gaëlle Tallet

Les premiers chrétiens et les signes du ciel François Bovon

Bar Kochba et les signes du Messie dans la littérature rabbinique

Christophe Batsch 
Les signes de la prophétie en Arabie à l'époque de Muhammad

(fin du $\mathrm{VI}^{\mathrm{e}}$ et début du $\mathrm{VII}^{\mathrm{e}}$ siècle de l'ère chrétienne)

Christian Julien Robin

\section{QUATRIÈME PARTIE}

\section{STATUTS ET LOGIQUES DU SIGNE}

Divination romaine et rationalité grecque dans la Rome du $\mathrm{II}^{\mathrm{e}}$ siècle avant notre ère

Jörg Rüpke

Fonction épistémologique du signe chez les Tannaïm et les

Amoraïm

Madalina Vârtejanu-Joubert

Du tirage au sort (qur'a) dans la loi islamique

Mohammed Hocine Benkheira

Les charaktêres, formes des dieux d'après les papyri et les gemmes magiques

Attilio Mastrocinque

Entre la nature et le rite: réflexions sur le statut des signes-voix divinatoires

Sabina Crippa

Les signes du futur dans le stoïcisme: problèmes logiques et philosophiques Jean-Baptiste Gourinat

Abstracts

Index rerum

Index nominum

601

Index locorum 608 


\title{
INTERPRÉTER LES SIGNES DU DIEU: UNE APPARITION DE MANDOULIS AU TEMPLE DE KALABCHAH*
}

\author{
Gaëlle Tallet
}

Converser «seul à seul» avec les dieux semble avoir été une préoccupation grandissante dans tout l'orient de l'Empire romain au cours des premiers siècles de notre ère. En effet, à l'époque impériale, des sources littéraires nous ont transmis des témoignages d'expériences spirituelles d'un type nouveau, reflétant le besoin de certains individus d'une rencontre personnelle avec le divin. Et c'est souvent vers l'Égypte qu'ils ont porté leurs pas: ainsi, le médecin Thessalos de Tralles, dans sa lettre-préface à des traités de botanique astrologique, rédigée au $\mathrm{I}^{\mathrm{er}}$ siècle de notre ère, relate le voyage qu'il entreprit dans un premier temps jusqu'à Alexandrie, pour perfectionner ses connaissances médicales, puis à travers l'Égypte et jusqu'à Diospolis (Thèbes) dans l'espoir d'apprendre des prêtres du lieu les mystères divins. Il parvint à gagner leur confiance et l'un d'eux céda à sa requête. Après un jeûne de trois jours, Thessalos se rendit dans une salle spécifique du temple: le prêtre «avait préparé une chambre (oikos) bien propre avec tout ce qu'il fallait pour la consultation; de mon côté, toujours prévoyant, j'avais apporté, sans le dire au prêtre, du papier et de l'encre pour prendre note, le cas échéant, de ce qui serait dit ${ }^{1}$.» Enfermé dans cette salle, Thessalos recevra la vision d'Asclépios (c'est-à-dire Imhotep), après s'être placé «face au trône». Cet épisode laisse penser qu'une salle d'un temple égyptien abritant une effigie divine (elles sont en général trônantes) pouvait être utilisée à l'époque impériale pour des consultations oraculaires privées, le privilège sacerdotal de voir le

* Une première version de cette présentation a fait l'objet d'une communication au séminaire interdoctoral «Religions et sociétés de la Méditerranée ancienne», à l'École Pratique des Hautes Études. Je tiens ici à remercier les participants et les organisateurs de ce séminaire, ainsi que les Professeurs Françoise Dunand et Jean-Marie Bertrand qui m'ont fait part de leurs suggestions.

1 Thessalos de Tralles, De Virtutibus herbarum 12-13 (traduction d'A. J. Festugière). Pour d'autres exemples de ce type, voir Festugière 1950, 45-66; Frankfurter 1998, 168-169 et 217-221; Dunand 2002. 
dieu "face à face» se démocratisant quelque peu. Pour rencontrer le dieu, Thessalos est prêt à parcourir l'Égypte jusqu'au Sud thébain.

C'est dans ce contexte qu'il faut probablement comprendre une série de textes en grec jadis peints en rouge sur les parois de la cour d'entrée, sur celles de la façade de la salle hypostyle, et sur les murs extérieurs du grand temple de Mandoulis à Kalabchah, en Nubie, à une cinquantaine de kilomètres d'Assouan (fig. 1) ${ }^{2}$. D'origine probablement nubienne, Mandoulis était une divinité locale inconnue hors du Dodécaschoène - territoire en amont d'Assouan qui correspond probablement à la Basse Nubie conquise par les Romains - et qui n'est pas attestée à l'époque pharaonique. La datation de ces textes s'étend $\mathrm{du} \mathrm{I}^{\mathrm{er}}$ siècle de notre ère à la première moitié du $\mathrm{III}^{\mathrm{e}}$ siècle ${ }^{3}$. Ils semblent concomitants de la présence d'une garnison romaine dans le bourg voisin de Talmis et couvrent une grande partie de la durée d'activité du temple. En effet, si la première installation cultuelle sur le site semble avoir été une chapelle ptolémaïque, la construction du grand temple lui-même a été entreprise sous Auguste et le bâtiment a été complété à l'époque de Vespasien; les décors pariétaux étaient toujours inachevés à l'époque antonine et la cour est restée sans décoration. L'activité du temple fut relativement brève: dans un décret daté de l'an 249 de notre ère, le gouverneur des nomes d'Ombos et d'Éléphantine, Aurelius Besarion, ordonne aux propriétaires de cochons de ne pas laisser leurs animaux entrer dans le sanctuaire, ce qui laisse supposer une désaffection des lieux ${ }^{4}$, et à la fin du $\mathrm{III}^{\mathrm{e}}$ siècle, la ville sortit complètement de l'influence romaine. Le temple fut ensuite transformé en église.

Édifié aux marges de l'Empire, sur un site isolé qui ne semble pas avoir été occupé avant l'époque ptolémaïque ${ }^{5}$, le temple devait se prêter à la rencontre spectaculaire du divin. Les nombreux graffiti de Kalabchah témoignent de l'importance des visites faites au dieu du sanctuaire par des hellénophones, qui y ont laissé des proscynèmes de

2 Sur le temple: Arnold 1975; Curto, Maragiglio \& Rinaldi 1965; Stock \& Siegler 1965. Voir aussi Habachi 1969; Rutherford 1998; enfin Kakosy 1964; 1978.

${ }^{3}$ Les proscynèmes datent du règne de Vespasien (69-79) jusqu'à celui de Sévère Alexandre (222-235). La dernière inscription datée de l'occupation romaine remonte à $248 / 249$, mais ce n'est pas un proscynème.

${ }^{4} S B, V, 8534=F H N$, III, 248. Toutefois, le culte continue alors à se dérouler dans le sanctuaire, puisque le décret ordonne d'évacuer les bêtes «pour que les rites sacrés puissent se dérouler selon la coutume».

${ }^{5}$ Griffiths 1929, 72; voir aussi Curto, Maragiglio \& Rinaldi 1965, 17, fig. 2. La vallée du Nil à cet endroit est étroite, rocailleuse et ne favorise pas l'installation de cultures.

(C) 2012 Koninklijke Brill NV 


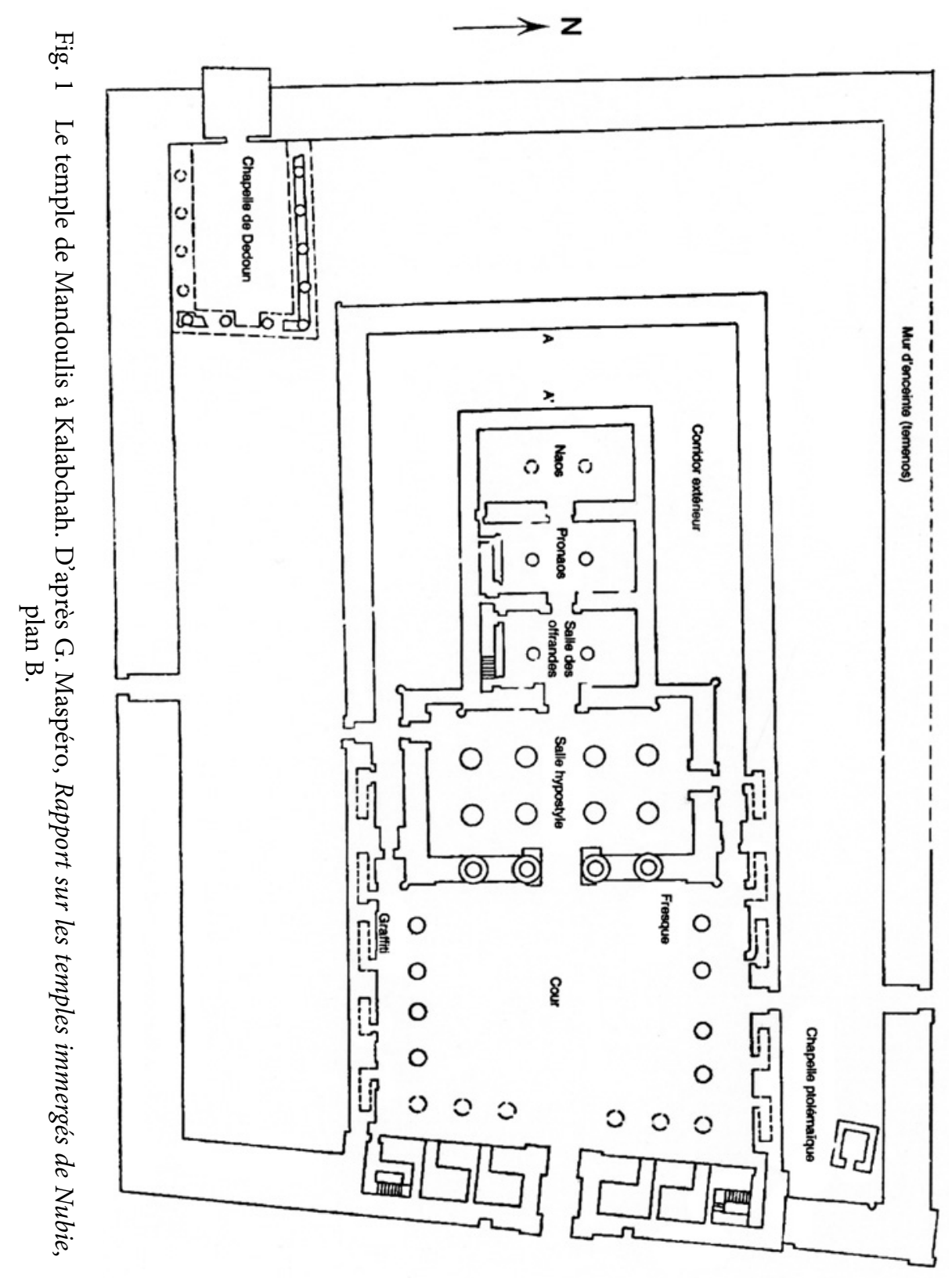

(C) 2012 Koninklijke Brill NV 


\begin{abstract}
AKTINOBONE $\triangle E C T O T A$ MANDOYAITITANMA: APEY CHMIACOYTINANAMITAOEAMENOC $\in \Pi \in N O H C A \omega A I E \Pi O A Y \Pi P A T M O C A A^{C} \phi \Delta \Lambda \omega C$ I $\triangle E N A I \theta \in \lambda \omega N E I C Y I O H \lambda I O C . \Delta \Lambda O T P I O N$ EMAYTONETOIHCAMHNTACHCI A EIAC

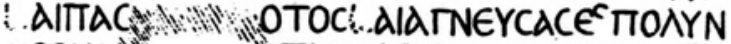
XPON : TIOEIACEYCEBIACIINEG. $\epsilon \pi \in \mathbb{R}$ D. AIENOEACAMENOCANE $N E Y W$ E $A E I Z A C M O I C E A Y T O N E N T W$ XPYCALII W: $\triangle \Phi O C \triangle I$ STPWNTATON

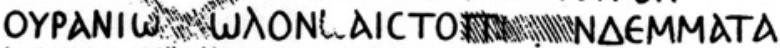
I ATA $\triangle$ EN NNYKTIAPOMON $\in N \omega^{\prime}$ : AIATIWTWTHCAOANACIACYAATIXOYCAMENOC
\end{abstract}

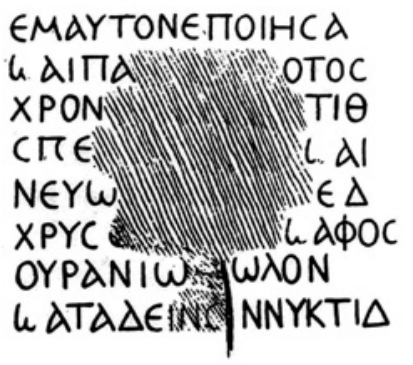

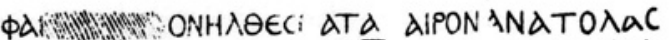
TIO!O EI EICTONCONCH' ONDDOANWTECW: AINAWEMTNOIAN TIPEXWN. ĀI Y NAMINMETANHNENOXCEETNWNMANDOYAI HAIONTONTIANTETIOTTHHNDECTOTHNATTANTWNBACI $\lambda E A$ 'AWNATIANTOKPATOPAWTWNEYTYXECTATWNNAWNTWN' ATOI' OYNTWN HNOHAOCMANAOYNICATATATHNIEPANTANMINHTICECTINYYTTO

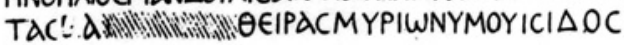

Fig. 2 Le relevé en trois parties de R. Lepsius (1844 par R. Lepsius). 
formulation relativement classique. Parmi eux, toutefois, se détachent cinq inscriptions dont la dimension «éthérée» a frappé les commentateurs $^{6}$ : elles sont écrites en vers et constituent de véritables petites pièces poétiques, avec des accumulations d'épithètes savantes et des allusions mythologiques à la manière alexandrine. Ces formes élaborées et développées du proscynème traditionnel laissent deviner l'itinéraire des visiteurs de Kalabchah, ou du moins de certains d'entre eux. En effet, comme le recommande une inscription laissée par un prêtre nommé Sansnos, ces personnages ont entrepris une visite au sanctuaire du dieu local, au cours de laquelle ils ont sollicité un signe du dieu, que ce soit dans le désir de mieux le connaître ou, plus communément, dans celui de lui demander d'intervenir en leur faveur. Le visiteur se mettait alors en condition pour pénétrer dans l'espace sacré, en échange de quoi il pouvait espérer obtenir une vision du dieu. Grâce à cette apparition, certains visiteurs accédèrent à la «véritable connaissance» et se promirent de glorifier Mandoulis, notamment au moyen de la rédaction d'un proscynème.

On constate une très nette parenté d'écriture, de style et de lexique entre ces cinq textes, dont l'un est acrostiche et livre le nom de son dédicant, le centurion Paccius Maximus ${ }^{7}$. Il est peu vraisemblable toutefois que Maximus ait dédié l'ensemble des cinq proscynèmes: dans l'un, c'est d'ailleurs un certain Hérodès qui demande à rentrer chez lui en bonne santé. Il y avait donc sur place à Kalabchah une personne qui rédigeait ces textes au profit de visiteurs soucieux de laisser leur trace dans le sanctuaire, et qui le faisait très vraisemblablement avec l'autorisation des prêtres du lieu, ce qui suppose un minimum d'organisation et d'encadrement des visites.

On peut donc distinguer deux groupes de textes qui diffèrent dans leur forme et leur contenu: à côté de proscynèmes courts tout à fait traditionnels, certains interrogent longuement la nature du dieu, allant pour l'un d'eux jusqu'à affirmer l'identité de Mandoulis avec l'Aiôn divin ${ }^{8}$. S'il est difficile d'imaginer l'existence de véritables pèlerinages, au sens actuel du terme, à Kalabchah, l'inscription de Sansnos nous incite à penser que les fonctionnaires en tournée dans la région, les hauts gradés inspectant les garnisons frontalières et, bien entendu, les

${ }^{6}$ Frankfurter 1998, 166.

${ }^{7}$ IM, 168; voir Burstein 1998.

${ }^{8} I M, 166$. 
soldats cantonnés à Talmis pouvaient profiter de l'occasion de leur séjour pour honorer le dieu local ${ }^{9}$. L'Égypte, patrie d'origine des dieux grecs pour Hérodote ${ }^{10}$, recelait, dans ses confins les plus barbares, l'espoir de la révélation divine ${ }^{11}$.

Dans le proscynème identifiant Mandoulis à l'Aiôn divin, inscrit dans la cour du temple sur le portique sud, les signes accordés par le dieu à son visiteur jouent un rôle tout à fait central ${ }^{12}$. Nous en donnons ici une lecture:

$S B, \mathrm{I}, 4127 ; I M, 166\left(\mathrm{II}^{\mathrm{e}}-\mathrm{III}^{\mathrm{e}}\right.$ siècle de notre ère $)$

$70^{\star} 102$

Talmis, temple de Kalabchah

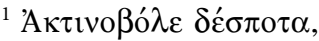

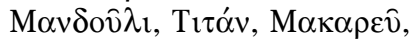

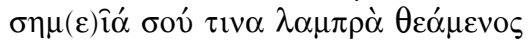

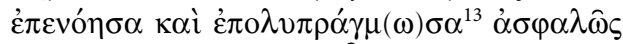

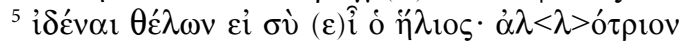

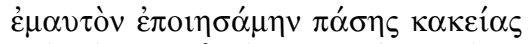

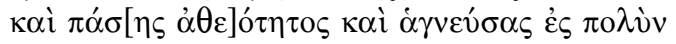

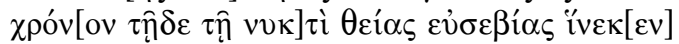

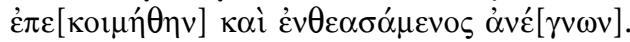

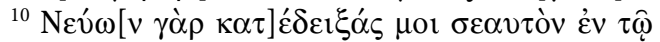

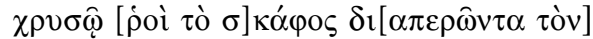

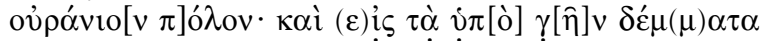

9 Sur cette question: Yoyotte 1960; Dunand 1997; Volokhine 1998. En l'occurrence, la très grande majorité des proscynèmes émanent d'un milieu militaire: leurs auteurs appartenaient à la Legio III Cyrenaica et à des corps auxiliaires. Parmi les civils potentiels, on peut citer un prêtre, Sansnos, un tailleur de pierre venu avec sa femme et son fils, et peut-être un médecin, qui pouvait aussi bien faire partie de l'armée. Il est possible également que les compagnes et les enfants des soldats aient été installés à proximité, comme c'est le cas dans d'autres établissements militaires.

10 «Presque tous les personnages divins sont venus en Grèce de l'Égypte. Qu'ils viennent de chez les Barbares, mes enquêtes me le font constater; et je pense que c'est surtout de l'Égypte» (Hérodote, II, 50). Voir aussi Platon, Phèdre 274c-275b.

${ }^{11}$ Nous sommes ici sur le territoire des redoutables Blemmyes, dont on peut voir une trace dans la "vision de Maximus»: Mandoulis descend de l'Olympe, «adoucissant le langage barbare des Éthiopiens» $(I M, 168,24)$.

${ }^{12}$ Le texte a été copié en août 1844 par R. Lepsius, sous la forme de trois fragments séparés (fig. 2). Lors de son passage à Kalabchah en janvier 1909, H. Gauthier en fit une révision et constata la disparition de nombreuses lettres depuis le passage de Lepsius, du fait de la montée des eaux du Nil après la construction du premier barrage. Les proscynèmes ont aujourd'hui disparu. Les éditions disponibles sont: Puchstein 1880, 71-74, $\mathrm{n}^{\text {os }}$ xxxiv B et C; Gauthier 1909; 1911, 241, n 3; Nock 1934; Lewy 1944; IM 166.

${ }_{13}^{13}$ Nous corrigeons le texte original: $\dot{\varepsilon} \pi \mathrm{o} \lambda \nu \pi \rho \alpha ́ \gamma \mu o \sigma \alpha$.

\section{(C) 2012 Koninklijke Brill NV}




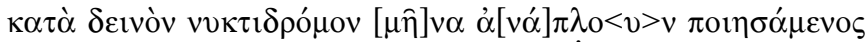

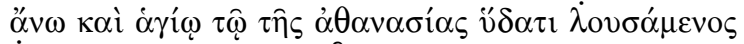

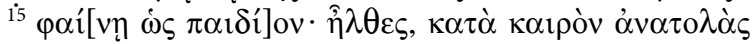

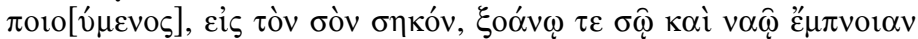

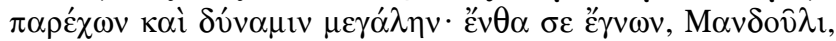

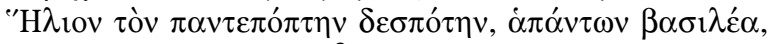

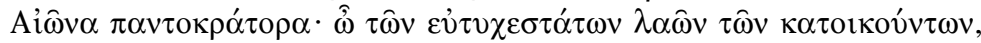

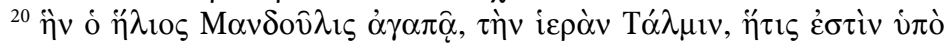

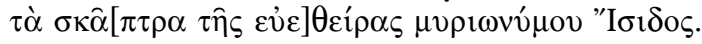

Seigneur qui lances tes rayons, Mandoulis, Titan, Makarée (?), contemplant tes signes éclatants, j'ai médité et recherché activement, dans le désir de savoir avec certitude si tu es bien le soleil. Je me suis rendu étranger à tout vice et à toute impiété, et, après être resté pur longtemps, cette nuit, à cause de ma piété envers les dieux, je me suis endormi, j'ai obtenu une vision et j'ai compris. En effet, accédant à ma prière, tu t'es montré à moi sur le flot d'or, faisant naviguer ta barque à travers la voûte du ciel. Et tu as remonté, dans ta barque, vers les formes qui sont sous terre, le cours nocturne de la terrible lune, tu t'es baigné dans l'eau sainte d'immortalité, et tu es apparu comme un enfant. Tu es venu en rayonnant au moment opportun, dans ton temple, donnant à ton image et au sanctuaire le souffle de la vie et une grande puissance. Alors je t'ai reconnu, Mandoulis, comme Hélios, le maître qui voit tout, le souverain universel, l'Aîon qui règne sur toutes choses. Peuples fortunés entre tous que ceux qui vivent dans la sainte Talmis que chérit le soleil Mandoulis et que régit le sceptre d'Isis aux belles boucles, aux mille noms!

La parenté de cet hymne avec la tradition oraculaire grecque apparaît nettement dans le choix de la forme poétique. Ainsi, on ne saurait parler ici d'hexamètres dactyliques, mais certains vers s'en rapprochent parfois, en empruntant des sections métriques de l'hexamètre; on notera également l'emploi de traits formels archaïsants, comme le génitif en - oto présent dans d'autres proscynèmes poétiques du dossier. Outre des images tout à fait classiques, on trouvera même à deux reprises dans ce groupe de textes un vers des Travaux et des jours $\mathrm{d}^{\prime} \mathrm{Hésiode}^{14}$. Mais, par sa thématique et sa forme, cet hymne oraculaire correspond à un type de requêtes qui se développent au $\mathrm{III}^{\mathrm{e}}$ siècle de notre ère dans les sanctuaires d'Asie Mineure et qu'Arthur D. Nock a qualifiées d' "oracles théologiques ${ }^{15}$ ».

14 Les travaux et les jours 91: IM, 167, v. 10; IM, 179, v. 10.

15 Nock 1928. 
Ainsi, un consultant du nom de Théophile adressa une requête similaire au dieu Apollon, préservée par la Théosophie dite de Tübingen ${ }^{16}$ :

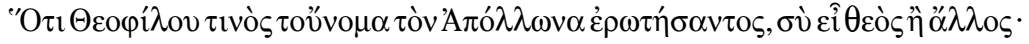

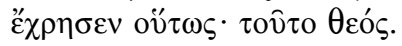

Comme un homme appelé Théophile interrogeait Apollon sur son nom, «Es-tu Dieu ou est-ce un autre?», il rendit cet oracle: «Tel est dieu ${ }^{17}$.»

Comme dans notre hymne, la réponse fera intervenir l'Aiôn divin, qui «était dans l'air du temps», pour reprendre une formule de Nock. On s'est longtemps interrogé sur l'authenticité des oracles rassemblés dans la Théosophie, étant donné les intentions apologétiques clairement affirmées de l'ouvrage, mais il est apparu, avec la publication par Louis Robert d'une inscription d'Oinoanda, en Lycie, contenant le texte d'une réponse oraculaire connue par ailleurs par la Théosophie et par Lactance, que certains pouvaient être des oracles authentiques, légèrement modifiés ou tronqués ${ }^{18}$. D'autres oracles de ce type nous sont conservés par les sources littéraires: ainsi, Macrobe a également transmis une consultation de nature théologique: «Qui est Iao?» ou «qu'est-ce que Iao ${ }^{19}$ ? "

Dans notre texte, le moteur, la cause de la venue du visiteur au

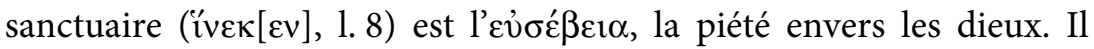
s'agit bien d'établir une relation personnelle avec la divinité. Ce visiteur, qui fait preuve d'un véritable empressement ( $\dot{\varepsilon} \pi \mathrm{o} \lambda \nu \pi \rho \alpha ́ \gamma \mu(\omega) \sigma \alpha$, 1. 4), se dit habité par son questionnement, par son désir ( $\theta \dot{\varepsilon} \lambda \omega v, 1.5)$ de connaître le dieu ( $\dot{\pi} \pi \varepsilon v o ́ \eta \sigma \alpha, 1.4)$. Cette volonté de «savoir avec cer-

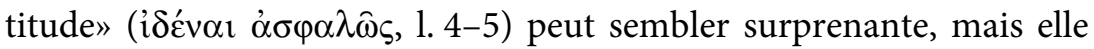
se rencontre également dans la démarche d'un néoplatonicien comme Jamblique, au $\mathrm{IV}^{\mathrm{e}}$ siècle $^{20}$ : au-delà de la connaissance rationnelle, c'est

${ }^{16}$ Cet ouvrage a été composé à l'extrême fin du v $\mathrm{v}^{\mathrm{e}}$ siècle de notre ère et consistait en un recueil d'oracles précédé d'une introduction en sept livres Sur la foi orthodoxe, la doctrine chrétienne. Nous utilisons pour ces textes la récente édition de P. F. Beatrice (Beatrice 2001); voir les considérations de Busine 2005, 2-7 et, pour les oracles de Claros, Merkelbach \& Stauber 1996.

17 Beatrice 2001, I, 2. T. L. Robinson a attribué à ce texte une origine clarienne, tandis que R. Lane-Fox privilégie l'oracle d'Apollon de Didyme.

${ }_{18}$ Robert 1971. On retrouve ce type d'interrogation dans d'autres oracles rapportés par la Théosophie: Beatrice 2001, I, 18.

19 Saturnales I, 18. Le même Macrobe raconte encore que Nicocréon de Chypre, à la fin du $\mathrm{IV}^{\mathrm{e}}$ siècle avant notre ère, aurait demandé à Sarapis quel dieu il était: Saturnales I, 20, 16.

${ }^{20}$ Carlier 1974, 257-263.

(C) 2012 Koninklijke Brill NV 
une expérience personnelle liée à une émotion, une révélation directe du dieu, qui est espérée. L'importance de la révélation est un des traits de la mutation de la sphère du sacré soulignée par Peter Brown dans l'Antiquité tardive: l'expérience religieuse se centre de plus en plus sur l'individu, conduisant des fidèles à rechercher activement, comme c'est le cas ici, la réclusion dans le temple afin d'avoir, par l'intermédiaire d'une pratique ascétique, une révélation de la divinitée ${ }^{21}$. Dès lors, la relation établie entre le dieu et son fidèle est marquée par une dimension hiérarchique et individualisée: Mandoulis est ici appelé

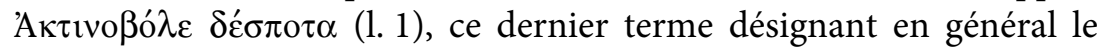
maître de maison, et dans d'autres proscynèmes, il apparaît comme un $\kappa \dot{\rho} \cos ^{22}$.

La consultation oraculaire est donc mise au service de la connaissance de la nature même du dieu. C'est une expérience mystique qui n'est pas seulement liée à une requête matérielle ${ }^{23}$ : les signes du dieu manifestent son identité et sa présence auprès du fidèle, et non plus quelque événement séculier à venir. Mais à la problématique de l'identité divine, présente à Claros ou à Didymes, vient s'ajouter ici une dimension de traduction proprement dite, dans la mesure où le dieu Mandoulis reste difficile à cerner pour un visiteur de culture grécoromaine. D'une certaine manière, ses signes devront être bilingues pour permettre une assimilation du dieu à des dieux gréco-romains connus, en l'occurrence Hélios, Apollon et l'Aiôn divin. Un des proscynèmes affirme d'ailleurs que la vision du dieu est destinée à assurer la traduction de son nom en grec $^{24}$ : en fait, notre visiteur demande au dieu de procéder lui-même à une interpretatio Graeca de son nom et de son image.

\section{La quête des signes}

Il se met donc dans la disposition nécessaire pour voir le dieu, en suivant un protocole de purification bien défini, indispensable à la fois pour pénétrer dans un sanctuaire égyptien (ce sont des dispositions auxquelles les prêtres sont déjà soumis à l'époque pharaonique)

21 Brown 1983, 21-63.

22 Ronchi 1974-1977, III, 622-625.

${ }^{23}$ C'est le cas de certains proscynèmes de notre corpus: IM, 167, 6-10; 169, 3-4.

${ }^{24}$ IM, 168, 17-18. 
et pour entrer en contact avec la divinité, vraisemblablement dans le cadre de l'incubation.

Il s'agit d'établir un sas avec la vie du monde, de «se rendre étranger» à tout ce qui caractérise la vie laïque quotidienne: $\dot{\alpha} \lambda(\lambda)$ ó $\tau \rho$ เov

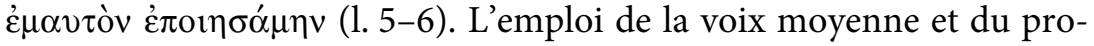
nom réfléchi, redondant, renforce l'idée d'un travail sur soi, d'une participation active du dédicant et pas seulement d'un rituel formel, ce qui là encore est caractéristique des formes de religiosité de l'Antiquité tardive. Le résultat de cette démarche est la pureté du candidat à la vision, peut-être liée à une chasteté rituelle et au port d'un vêtement

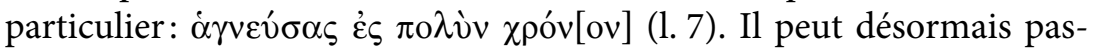
ser la nuit dans le temple. Deux restitutions ont été proposées pour le passage qui suit, très corrompu.

Tout d'abord, Hans Lewy, suivi par Étienne Bernand, a proposé de

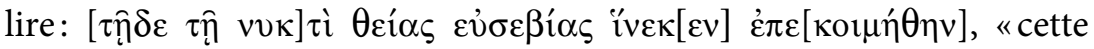
nuit-là, à cause de ma piété envers les dieux, je me suis endormi (pour obtenir une vision)».

Le verbe $\dot{\varepsilon} \pi \varepsilon[\kappa o น \mu \eta \eta \eta \eta v]$ est restitué d'après le terme ordinairement

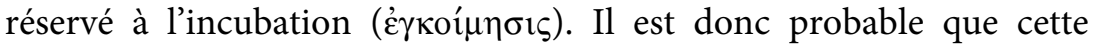
lacune désignait, comme c'est habituel, le moment même de la vision,

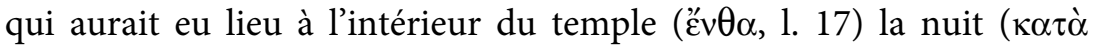

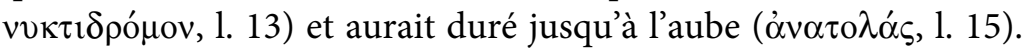

Toutefois, Arthur D. Nock fait une autre suggestion et lit: $\alpha \gamma v \varepsilon v ́ \sigma \alpha \varsigma$

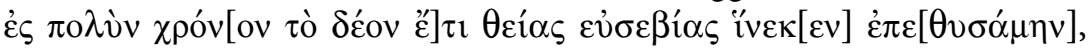
«je fus chaste pendant une période importante et fis les offrandes d'encens comme il convient, par divine piété $»^{25}$.

Cette restitution coïncide mieux avec l'état du texte. L'offrande d'encens est préalable à tout acte rituel et vient compléter ici la purification entreprise dans les vers précédents. La vision ne serait pas dans ce cas obtenue en songe, dans le cadre d'une incubation, mais à l'état de veille.

À l'issue de cette purification, le dédicant obtient une vision: le terme $\dot{\varepsilon} v \theta \varepsilon \alpha \sigma \alpha ́ \mu \varepsilon v o \varsigma$ (l. 9) renvoie à l'idée de transport, d'inspiration et au type de la divination dite inspirée, mais le mystère même de l'objet de cette vision est maintenu.

${ }_{25}$ A. D. Nock rapproche ce passage du texte de Plutarque, De Iside $₫ 52$, Moralia 372 C-D.

(C) 2012 Koninklijke Brill NV 
Certes, ce passage est extrêmement rhétorique: à la manière alexandrine, on privilégie ici des termes abstraits, comme $\kappa \alpha \kappa(\varepsilon) \hat{\imath} \alpha$ (mal) et peut-être $\alpha \dot{\alpha} \theta$ có $\eta$ s (impiété, éloignement des dieux). Toutefois, une pratique effective est probablement à la source de cette composition. Il était devenu courant en Égypte à l'époque impériale de poser des questions à un oracle, de chercher à obtenir des rêves prophétiques ou de dormir dans les temples pour obtenir des rêves indiquant des moyens de guérison ${ }^{26}$. Qu'il s'agisse de séances d'incubation dans un temple ou de rituels magiques privés, le jeûne, la purification de soi par de l'encens ou par l'abstinence jouent un rôle prépondérant. Par ailleurs, un autre texte du corpus de Kalabchah laisse penser qu'un oracle par incubation y fonctionnait: dans le texte désigné comme la "vision de Paccius Maximus", ce dernier dit avoir reçu des consignes du dieu pendant un songe, alors qu'il était descendu dans un «souterrain propice au sommeil (ïnvov $\left.\mu \nu \chi \sigma_{\varsigma}\right)^{27} »$. L'expression, a-t-on pensé, pouvait désigner une pièce en sous-sol, à l'intérieur du temple, destinée à l'incubation - il se trouve qu'un mammisi rupestre, dans l'enceinte du temenos de Kalabchah, aurait pu jouer un tel rôle. Mais il est probable qu'il n'y a pas eu de lieu spécifique destiné au sommeil. Le terme de $\mu \nu \chi o ́ s$, employé également dans le cadre de l'oracle de Delphes, pourrait désigner une partie du temple légèrement plus basse que le niveau de l'édifice ou dissimulée aux regards, mais pas nécessairement un lieu souterrain. Si le $\mu \nu \chi o ́ \varsigma$ doit être un souterrain, peut-être renvoie-t-il plutôt au contenu du rêve incubatoire de certains pèlerins: notre hymne semble faire allusion à l'espace que le soleil traverse dans sa course nocturne.

Ce pouvoir curatif et sacré du lieu même du temple n'est pas inconnu dans les sources égyptiennes de l'époque pharaonique, mais il y a lieu de penser que l'incubation n'était pas le mode de consultation oraculaire le plus répandu et qu'il était réservé à la classe sacerdotale. C'est à l'époque gréco-romaine surtout que se développe ce type de pratiques attestées dès le début de la période ptolémaïque sur la terrasse supérieure du temple funéraire d'Hatchepsout à Deir el-Bahari, avec les cultes des dieux guérisseurs Amenhotep, fils de Hapou, et Imhotep, ou dans le contexte plus hellénisé du Serapeum de Canope. L'incubation à grande échelle semble donc à bien des égards être un développement

26 Sauneron 1959; Assmann 1978; Frankfurter 1998, chap. 4.

${ }^{27}$ IM, 168, 7-8. 
hellénistique de l'oracle égyptien. Dans le cas qui nous intéresse, ses modalités sont tout à fait classiques: le signe qu'accorde le dieu est ici une vision, en rêve ou en état de veille, sous l'effet de l'abstinence et de la fatigue. C'est un signe qui intervient dans un cadre technique et nécessite vraisemblablement l'intervention de spécialistes autorisés à en donner l'explication.

\section{La construction du signe divin}

Les signes du dieu que le fidèle vient rechercher ici sont donc l'objet d'un encadrement au sein du processus de consultation oraculaire, encadrement dont témoigne la construction même de l'hymne. En effet, au cours de cette expérience et au fil des vers qui la relatent, le signe divin est investi de contenus différents et progressivement

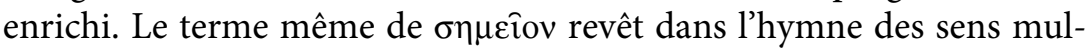
tiples qui impliquent la mise en place de différents niveaux de lecture et correspondent à autant d'étapes du cheminement du visiteur vers la "véritable connaissance» du dieu. Ce cheminement comporte, selon nous, trois moments, qui sont mis en relation avec trois sens et trois expériences du $\sigma \eta \mu \varepsilon i ̂ o v$ divin.

\section{A. Le signe comme lieu d'identification}

$\mathrm{Au}$ seuil de sa rencontre avec le dieu, notre visiteur affirme avoir

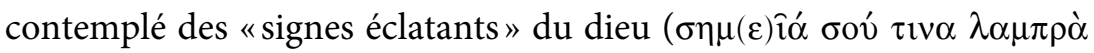
$\theta \varepsilon \alpha ́ \mu \varepsilon v o \varsigma, 1.3)$, qu'il n'a pas tout de suite compris. Mais de quels «signes» peut-il bien s'agir? Le texte maintient ici une ambiguïté manifeste.

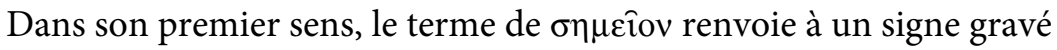
ou écrit ${ }^{28}$. Les "signes de Mandoulis» sont donc peut-être à comprendre, en premier lieu, dans le sens de «représentations» figurées ou éventuellement textuelles du dieu ${ }^{29}$. Ces signes sont "éclatants", $\lambda \alpha \mu \pi \rho \alpha$, c'est-à-dire d'un éclat semblable à celui du soleil ou d'un astre $^{30}$. Mais, à cette étape de l'expérience, le dédicant n'a pas acquis

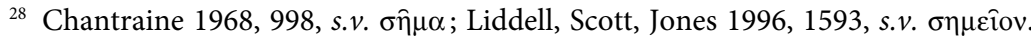

${ }^{29}$ L'écriture hiéroglyphique des textes pariétaux du temple pouvait être perçue comme telle.

${ }^{30}$ On l'utilise en parlant du soleil (Iliade I, 105), de la lune, des astres, du jour, de l'éclat

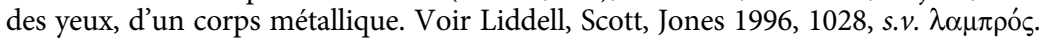

\section{(C) 2012 Koninklijke Brill NV}


la certitude qu'il a affaire au dieu du soleil. L'éclat qui l'a frappé dans un premier temps est peut-être celui des représentations dorées du temple, dont témoignent les notes d'Henri Gauthier. En effet, lors de son passage, un enduit doré était encore en place en divers endroits du sanctuaire et les premiers voyageurs parlent de Kalabchah comme d'un temple doré. Entre l'éclat du soleil et celui de l'or du temple, le texte maintient ici une subtile ambiguïté que la splendeur solennelle du sanctuaire isolé au milieu du désert devait contribuer à rendre particulièrement intense. Au fil de l'expérience de la vision du dieu, le

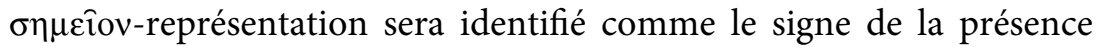
du dieu et deviendra éclatant, c'est-à-dire également «évident», clair, limpide pour le fidèle, lumineux comme le soleil et comme l'expérience épiphanique.

Cependant, avant d'atteindre cette ultime étape de compréhension des signes du dieu, avant d'identifier ce à quoi ils renvoient, le visiteur reste perplexe et les $\sigma \eta \mu \varepsilon i \alpha$ du début de l'hymne constituent autant d'indices à déchiffrer dans l'enquête qu'il va mener pour identifier «avec certitude» l'hôte de Kalabchah. Le regard que le visiteur porte sur ces représentations est marqué à la fois par l'étonnement admiratif et par l'examen ${ }^{31}$.

De cette hésitation entre deux statuts du $\sigma \eta \mu \varepsilon i o v$, représentation du dieu ou signe de sa présence, les textes égyptiens rendent bien compte. En effet, dans la conception égyptienne, l'image divine est considérée comme un corps terrestre du dieu et, comme son (ses) nom(s), elle fait partie des éléments définitionnels de son identité. Mais les textes des temples égyptiens distinguent différents types d'images, plus ou moins investies de la présence divine. Comme le note Claude Traunecker dans une contribution sur l'image égyptienne et la production du sacré, l'image peut être un simple support de signification et correspondre à une sorte de signe hiéroglyphique chargé de transmettre une information; c'est le cas par exemple des décors pariétaux et, tout particulièrement, des décors des murs d'enceinte (images-sededj).

Cette première catégorie d'images peut être le signe, la marque de reconnaissance qui permettra à l'entité divine, quand elle parcourt les cieux, d'identifier sa demeure terrestre, le temple ${ }^{32}$. Ainsi, selon un bandeau du temple d'Edfou, le dieu Horus «étend les ailes, il découvre

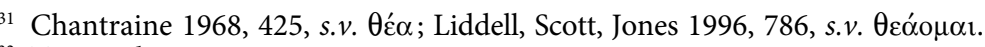

32 Traunecker 1991, 84. 
son image (sededj) sur son mur d'enceinte. Il se pose dans sa maison, il s'unit à son temple. Il embrasse son image (bes) dans son $n a o s^{33}$ ». Le passage distingue nettement l'image pariétale (image-sedjedj) de la statue de culte (image-bes) qui est une image «initiée» et possède un degré d' "animation" plus élevé. La première est la marque de l'identité du monument et matérialise l'attribution d'un espace cultuel à la divinité, tandis que la seconde est le réceptacle véritable de la présence divine. Dans ce dernier cas, l'image et l'entité représentée sont confondues ${ }^{34}$.

La démarche du visiteur de Kalabchah s'apparente à notre sens à celle d'Horus identifiant son sanctuaire grâce aux images divines de son mur d'enceinte: c'est là un premier sens des $\sigma \eta \mu(\varepsilon) \hat{\imath} \alpha$ qu'évoque

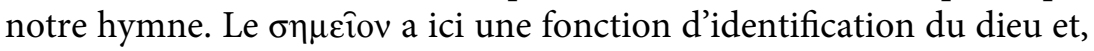
comme les images des décors pariétaux, sert à rappeler qui est le dieu principal et à indiquer sa demeure.

La perplexité du visiteur, au départ incapable d'identifier le dieu grâce à ses images, trouve une réponse au cours de la consultation oraculaire, probablement sous la forme d'une «catéchèse égyptienne " délivrée par les prêtres du sanctuaire. Cet encadrement sacerdotal intervient en effet, nous semble-t-il, dans l'explicitation de la nature de Mandoulis, qui ne pouvait apparaître de manière évidente aux yeux d'un Grec ou d'un Romain comme un équivalent d'un dieu solaire grec. Sa théologie est complexe et le dieu, double, est difficile à appréhender: il est désigné d'une part comme "Mandoulis le grand dieu » et se présente alors sous une forme adulte, le plus souvent coiffé du diadème atef; d'autre part, il apparaît sous la forme de "Mandoulis l'enfant», avec la tresse de l'enfance et le doigt à la bouche, coiffé du diadème hemhem ${ }^{35}$. Il appartient, d'une part, à la famille osirienne: placé dans la continuité du roi défunt Osiris, il assume, à l'instar d'Harendotès (Horus protecteur de son père), le rôle de vengeur de son père et de restaurateur de l'ordre de la royauté. D'autre part, il assume un rôle de dieu solaire, ce qui n'est pas étonnant si l'on considère l'articulation qui s'effectue de plus en plus nettement en Égypte, à partir du Nouvel Empire, entre deux représentations de la royauté, osirienne

${ }^{33}$ Edfou I, 328, n ${ }^{\text {os }} 10-11$.

${ }^{34}$ Voir aussi Bernand 1988.

${ }^{35}$ Le saint des saints porte les traces d'un naos double abritant les deux statues de la divinité et, dans l'ensemble du temple, le dédoublement de Mandoulis se décline à l'envi.

(C) 2012 Koninklijke Brill NV 


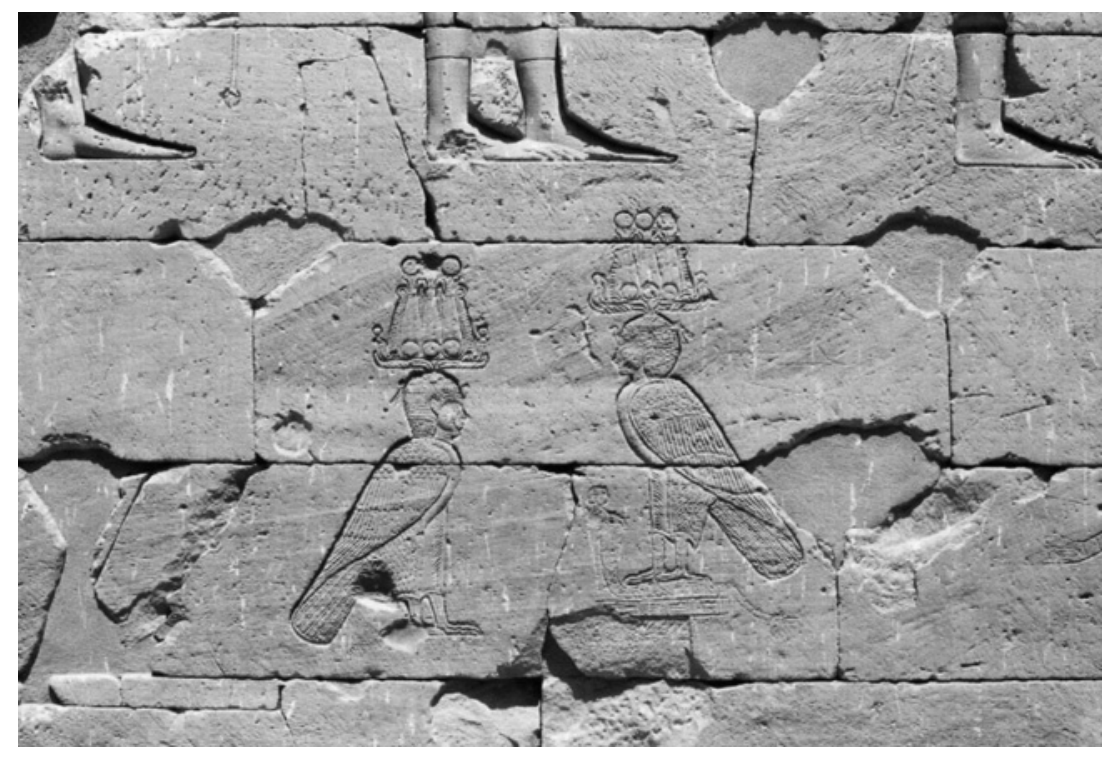

Fig. 3 Graffiti de visiteurs à Kalabchah. D’après Hölbl 2004, 130, fig. 189.

et solaire. Mandoulis est donc à la fois une image du pouvoir royal et de sa transmission, une figure dédoublée entre un détenteur du pouvoir et un héritier, et une figuration des étapes du cycle solaire, soleil à son zénith (le grand dieu, netjer $a a$ ) ou force renaissante du soleil (l'enfant, pa shered). Il y a une interpénétration très forte entre les deux formes du dieu adulte et du dieu enfant et de nombreuses confusions iconographiques et onomastiques, peut-être délibérées, qui s'accroissent à mesure qu'on s'éloigne du cœur du sanctuaire, rendent la compréhension de Mandoulis délicate et justifient donc la requête du dédicant. Toutefois, malgré cette complexité, certains visiteurs avaient manifestement connaissance de la double nature du dieu de Kalabchah, comme en témoignent des graffiti représentant deux formes du dieu (fig. 3$)^{36}$.

Différentes scènes du temple ont pu servir de support à une explicitation de la personnalité de Mandoulis par les prêtres du lieu. L'une d'elles orne le linteau intérieur du pylône d'entrée du temple, dans la cour à ciel ouvert accessible à tous les visiteurs, et l'autre se trouve dans

\footnotetext{
${ }^{36}$ Par exemple, sur l'entrecolonnement de la façade de la salle hypostyle: Gauthier 1911, pl. LXXI.
} 
le couloir extérieur qui entoure le naos du dieu proprement dit, dont les graffiti de pèlerins attestent qu'il était en partie accessible. Mais c'est surtout une installation tardive qui nous incite à penser qu'une chapelle adossée fonctionnait dans le couloir extérieur, à l'arrière du naos (fig. 4). Ces chapelles jouaient un rôle central dans la diffusion par le clergé des images divines, dans un véritable jeu de mise en scène et d'épiphanie des dieux. En l'occurrence, ces deux représentations de Mandoulis, qui ornent le centre du mur ouest du déambulatoire, sont sculptées sur quatre assises de pierre aplanies et dominées par une entaille profonde qui devait recevoir un auvent. Des entailles à la base laissent supposer qu'un plancher de bois avait été aménagé pour compléter la petite chapelle. Certaines traces de chevilles permettent d'imaginer un décor rehaussé d'une plaque d'or ${ }^{37}$. Il est très vraisemblable que c'est à ce type d'images de Mandoulis qu'a eu accès, dans un premier temps, notre visiteur: ces images étaient dorées, éclatantes. Le tableau que pouvait contempler le dédicant présente deux images de Mandoulis face à face ${ }^{38}$. Les prêtres disposaient là d'un véritable manuel de théologie simplifiée pour diffuser les traditions du temple, comme c'était aussi le cas à Kom Ombo.

Partant d'un tel support et de probables explications du personnel

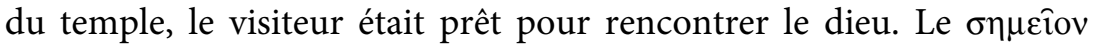
comme représentation n'est donc qu'un préalable à la prise de signes techniques qui suit: une distinction est mise en place entre ce que l'image du dieu laisse entrevoir au néophyte et ce qu'une herméneutique appropriée permet d'atteindre à travers cette même image, faisant d'elle un signe de la présence divine. La séance d'incubation, qui suscite une vision en rêve et sera aussi interprétée par des prêtres, transforme les signes-représentation en «signes de puissance».

${ }^{37}$ Ce type de placage est connu dans d'autres sanctuaires de l'époque grécoromaine, en particulier à Dendéra où l'arrière du naos était orné d'une immense face dorée d'Hathor. Voir aussi les dorures associées au bâtiment du contre-temple à Kellis: Hope et alii 1989, 15.

${ }^{38}$ Ce dispositif n'était vraisemblablement pas encore en place à l'époque où notre texte a été inscrit: selon C. Desroches-Noblecourt 1985, cette scène est juste antérieure ou contemporaine du règne de Silko, au vi ${ }^{\mathrm{e}}$ siècle de notre ère. Auparavant, d'autres représentations du déambulatoire pouvaient jouer le même rôle, de manière plus rudimentaire: c'est le cas d'une autre scène qui ornait l'arrière du naos et qui rappelle les deux dieux dont les statues étaient installées côte à côte dans le naos, entourés de leur famille divine. 


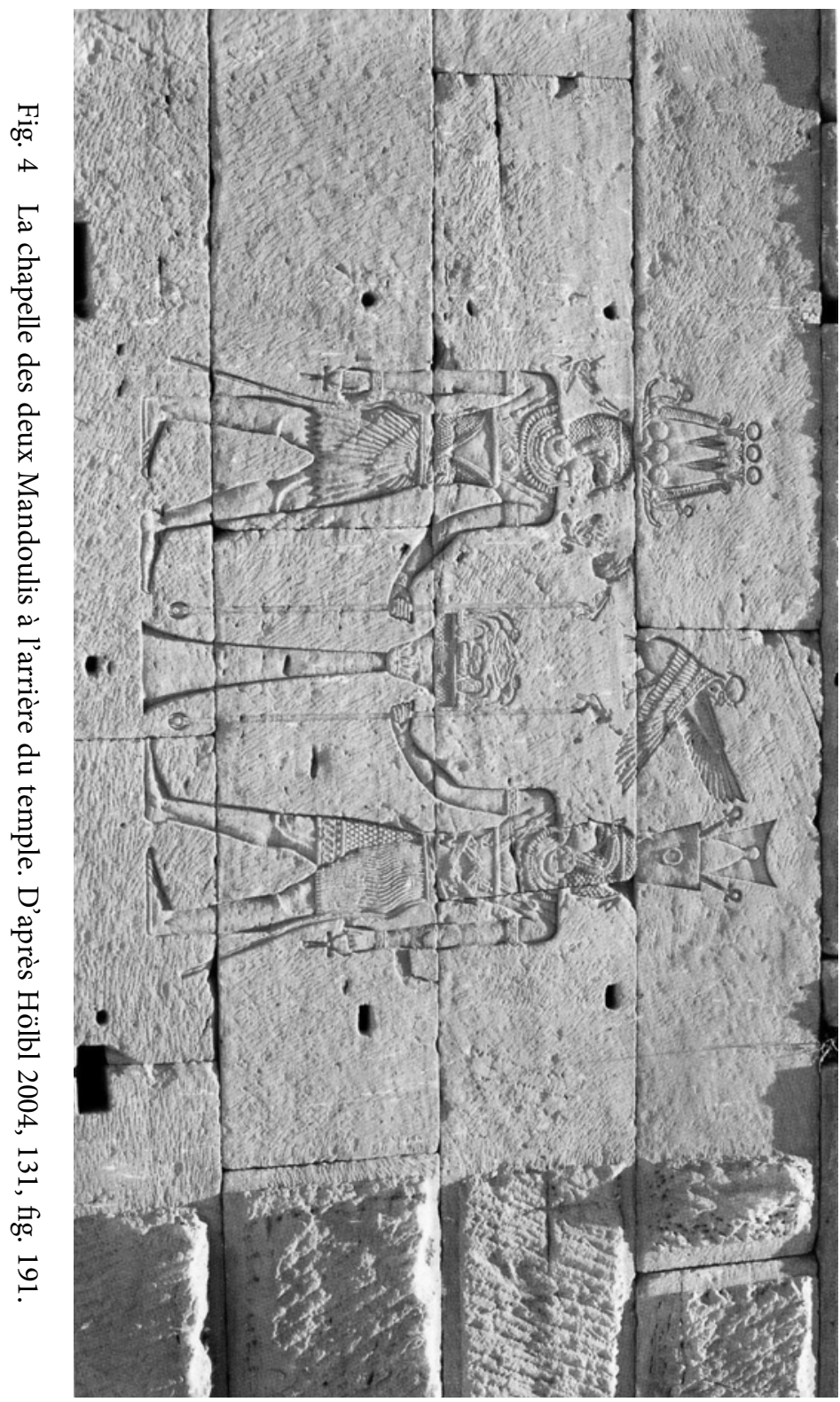

(C) 2012 Koninklijke Brill NV 


\section{B. Le signe comme lieu de puissance}

Comme nous l'avons mentionné plus haut, les images divines égyptiennes n'ont pas seulement une fonction d'évocation ou d'identification des entités divines. Qu'il s'agisse d'une statue cultuelle, d'un bas-relief de temple, d'une statuette fruste de terre cuite ou bien encore de l'animal considéré comme l'hypostase d'un dieu, comme sa statue vivante, une effigie peut remplacer le corps d'un dieu ${ }^{39}$. Elle possède, une fois accomplis les rituels appropriés d'animation de l'effigie, les mêmes qualités, la même intensité de réalité, d'existence, que l'être dont elle est le signe ${ }^{40}$.

L'image cultuelle - le Goóvov mentionné plus loin dans l'hymne (1. 16) ${ }^{41}$ - matérialise le dieu sur terre et appartient par excellence à cette dernière catégorie: lorsqu'elle a subi l'opération spécifique de l'«ouverture de la bouche» et le rituel de l'animation, son statut relève d'une réalité objective admise par tous ${ }^{42}$. Elle fait partie de ce que Claude Traunecker appelle les «images d'action ", c'est-à-dire les statues et images de culte habitées par le $b a$ divin, qui sont des lieux de hiérophanie et dont la conservation et l'entretien justifient l'existence même du temple. Toutefois, toute image divine participe du corps du dieu et les images pariétales sont parfois désignées comme des imagesbes ou encore comme des «images de puissance» (sehemou) ${ }^{43}$. C'est probablement face à ces images pariétales, ou à celles de la chapelle adossée - et pas nécessairement face à la statue cultuelle elle-même -, que le visiteur prend conscience de l'animation des représentations du dieu, de la présence divine dont elles sont intensément le signe.

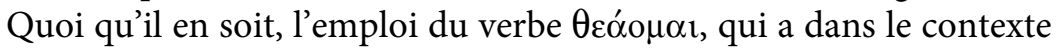
théâtral le sens très précis de "voir en tant que spectateur", renvoie à une dimension bien particulière de l'expérience religieuse que ménage

${ }^{39}$ Comme le rappelle Philippe Derchain, dans son analyse du PSalt. 825, dans les rituels, un hippopotame de cire ou de gâteau peut tenir lieu d'hippopotame réel, et il est considéré comme le point d'émergence de la force divine, maléfique dans ce cas précis: Derchain, 1965, 9; Säve-Söderbergh 1953, 43.

${ }^{40}$ Hornung 1996, 166.

${ }^{41}$ Le terme qui désigne en général, dans les inscriptions et papyrus d'Égypte, des images processionnelles: cf. Preisigke, Wb III 382; OGIS, 90, 107.

${ }_{42}$ Ainsi, à Edfou, deux textes gravés sur la frise extérieure de la salle hypostyle décrivent les rites de consécration du temple: après avoir effectué la série complète des opérations du rituel de l'ouverture de la bouche, probablement sur les statues de culte, on assure l' "ouverture de la bouche» du temple lui-même, considéré comme un objet de culte. Voir Blackman \& Fairman 1942; Edfou IV, 330-331.

${ }_{43}$ Traunecker $1979 ; 1991,88-92$.

\section{(C) 2012 Koninklijke Brill NV}




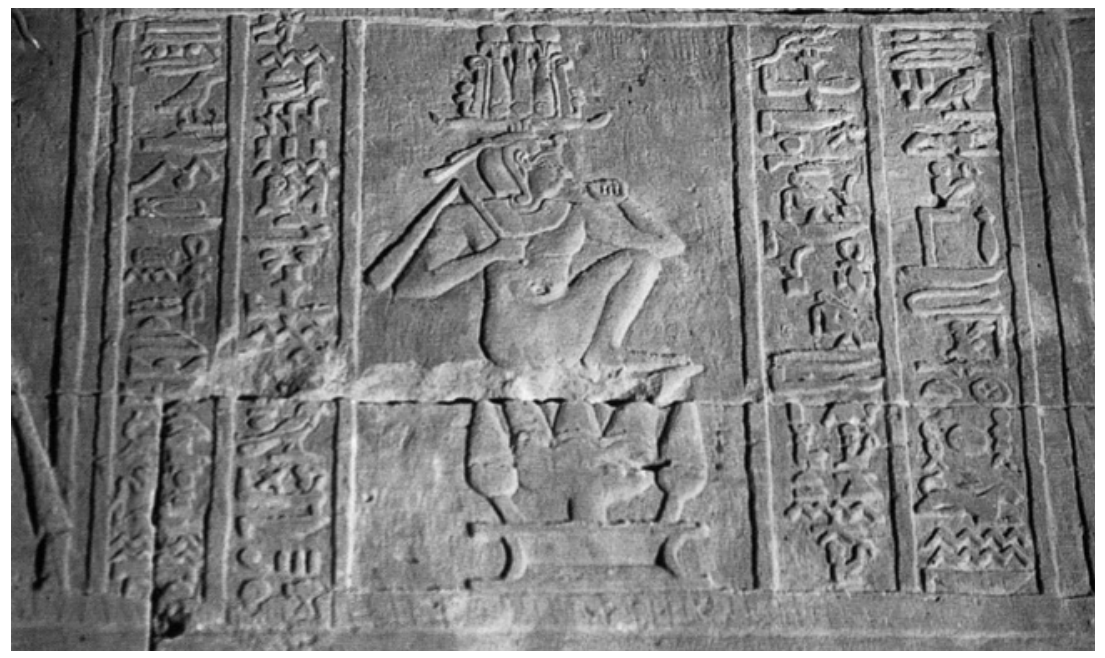

Fig. 5 Mandoulis l'enfant dans le pronaos de Kalabchah. D'après Hölbl 2004, 126, fig. 180.

la religion égyptienne. La notion de spectacle est tout à fait fondamentale dans le contexte du temple égyptien et de la transmission du savoir aux «laïcs»: la fête égyptienne, occasion exceptionnelle de contact entre le monde du dieu et les fidèles, se dit en effet $k h \hat{a}$, «apparition", et culmine sur l'apparition processionnelle, en public, de la statue divine ${ }^{44}$. Nous y voyons l'indice d'une mise en scène spectaculaire de l'image ou de la statue divine par le clergé du temple.

Un dispositif architectural du pronaos pouvait permettre une telle mise en scène: la fenêtre taillée au sommet du mur nord surmonte une scène où l'enfant solaire est accroupi au-dessus d'un lotus flanqué de deux boutons, émergeant du bassin primordial. À deux moments de la journée, le rayonnement du soleil venait illuminer ce tableau et l'inscription qui l'encadre, inscription qui affirme l'apparition sans cesse renouvelée de Mandoulis (fig. 5).

C'est, semble-t-il, à cette vision que se réfère l'évocation de Mandoulis dans l'hymne comme un dieu rayonnant:

${ }^{44}$ L'importance prise par cette dimension spectaculaire à l'époque impériale a été soulignée par D. Frankfurter: Frankfurter 1998, 58-60. Dans les processions, les naoi portatifs étaient souvent munis de portes partielles permettant de jouer sur le visible et le caché. Voir sur cette question Van den Plas 1989. 


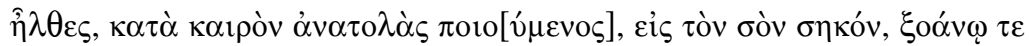

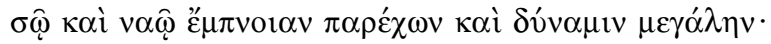

Tu es venu en rayonnant au moment opportun, dans ton temple, donnant à ton image et au sanctuaire le souffle de la vie et une grande puissance.

Répondant à l'appel du visiteur, Mandoulis se lève donc, rayonnant, dans toute sa splendeur solaire. L'apparition du soleil au petit matin

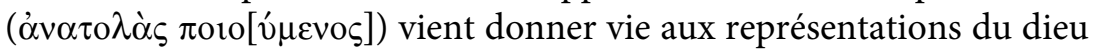
dans le pronaos. Ce sont donc les signes de la présence du dieu, les rayons du soleil, sorte de statue vivante du dieu soleil ${ }^{45}$, qui viennent animer les signes-représentations (les représentations figurées du dieu), qu'il s'agisse des reliefs pariétaux ou de la statue de culte du dieu.

Notre passage évoque à bien des égards un rituel qui se déroulait chaque matin dans le temple et qui était en principe réservé au prêtre stoliste: ce prêtre, seul à avoir le privilège de "voir le dieu en face», ouvrait le naos totalement fermé et obscur de la statue du dieu pour l'éveiller à la vie au contact des rayons du soleil. Ce sont ces vertus régénérantes du soleil que captaient également des cérémonies annuelles - ou plus récurrentes - désignées sous le nom d'Union au Disque. François Daumas a montré qu'il y avait sur le toit du temple de Kalabchah, accessible par un escalier, une chapelle pure, située à un endroit de la terrasse correspondant au fond du sanctuaire et protégé des regards profanes ${ }^{46}$. C'est probablement en cet endroit qu'était célébré le rituel de l'Union au Disque ${ }^{47}$. À Edfou, Dendara ou el-Qal'a, c'était en tout cas dans cette chapelle que, la veille du Nouvel An, la statue divine était portée, ointe et parée pour la grande cérémonie de l'aube, où elle était exposée à la lumière du soleil et rechargée en énergie nouvelle, au moment périlleux pour l'équilibre cosmique que représente le passage à la nouvelle année, lors de la terrible «nuit de Rê». L'itinéraire de la procession, en circuit fermé, évoquait celui du soleil parcourant sur sa barque le corps de la déesse du ciel, Nout.

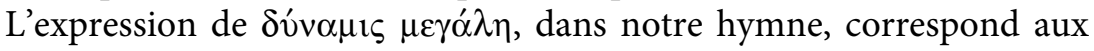
fonctions de cette cérémonie: l'âme du dieu rejoint son support terrestre en une union mystique qui assure pour une année le maintien

\footnotetext{
${ }^{45}$ De même que les animaux sacrés sont perçus en Égypte comme des statues vivantes des dieux, l'obélisque est compris comme une pétrification du rayon solaire.

${ }^{46}$ Daumas 1970.

${ }^{47}$ L'escalier de Kalabchah, inachevé, ne porte pas d'inscription qui puisse expliciter sa fonction, mais son organisation et celle du toit vont dans ce sens.
} 
de la fertilité et de la prospérité. Tous les temples d'Égypte célébraient simultanément cette fête considérée comme vitale pour le pays.

Notre visiteur aurait-il assisté à une telle cérémonie? Le terme de Góavov, au sens de statue processionnelle, peut le laisser penser. Toutefois, l'exiguité de l'escalier et de la chapelle ne permettait probablement pas à de nombreuses personnes d'assister à la cérémonie et, à Edfou, la Fête du Nouvel An, connue par les représentations ornant l'escalier qui menait au toit, était interdite aux profanes. Toutefois, d'après Maurice Alliot, à Edfou, l'Union au Disque n'était pas seulement célébrée lors du Nouvel An, mais en différentes occasions solennelles, et les rites pouvaient avoir lieu soit dans la ouabet (chapelle pure) d'en bas, soit sur les terrasses du temple. Et peut-être un accès au moins visuel était-il possible: dans le temple d'el-Qal'a, d'époque impériale, un système de portes se fermant en deux parties et pouvant fonctionner comme des barrières, a été mis en valeur par Claude Traunecker; en certaines occasions, des espaces du temple habituellement clos pouvaient être vus par des visiteurs extérieurs triés sur le volet, sans qu'on puisse y entrer ${ }^{48}$.

Cette hypothèse est étayée par la mention du "moment opportun" ( $\kappa \tau \tau \grave{\alpha} \kappa \alpha i \rho o ́ v)$, qui évoque des cérémonies de ce type, attestées au Serapeum d'Alexandrie ${ }^{49}$. Grâce à de savants calculs, dans ce sanctuaire, les rayons du soleil venaient illuminer la statue au fond de son naos lors de fêtes annuelles (ou bi-annuelles). Au jour précis de la venue du visiteur de Kalabchah, le soleil se serait levé dans l'axe du sanctuaire, illuminant la statue de culte qui se trouvait dans le naos, ou bien il aurait éclairé les représentations pariétales du pronaos par la fenêtre mentionnée plus haut.

En tout cas, le lexique utilisé ici pourrait tout à fait renvoyer à un discours originel égyptien, adapté en grec par des prêtres égyptiens, tant certains textes d'Edfou évoquent dans les mêmes termes le lever d'Horus-Rê lors de la Fête du Couronnement ${ }^{50}$. Témoin également cet hymne proclamé sous la fenêtre-soupirail de l'escalier conduisant à la terrasse du temple d'Edfou, au cours du rituel d'Union au Disque des statues d'Horus et d'Hathor lors des Fêtes du Siège de la première fête:

${ }^{48}$ Traunecker 1997, 176.

${ }^{49}$ Thélamon 1974.

${ }^{50}$ Voir par exemple Alliot 1954-1959, II, 647.

(C) 2012 Koninklijke Brill NV 
Le soleil est apparu du côté de l'orient: la voûte céleste est débarrassée des nuages. Horus de l'Orient brille au ciel, il entre au soupirail du Grand siège. C'est l'âme de l'Orient qui s'élève vers les cieux, et fait resplendir sa lumière par la fenêtre! L'Horus des Horus se lève; il s'éloigne vers le ciel, unissant ses rayons à son image sainte: c'est le dieu matinal, c'est Khépri (qui sort) du monde inférieur à l'aube, et dont l'éclat [...]! Râ se manifeste sur la montagne de l'Est, s'élevant au firmament; sa splendeur illumine le Siège des deux dieux ${ }^{51}$ !

Mais à la même époque, dans les contextes rituels du monde grécoromain, le rayonnement solaire faisait également sens. Dans l'oracle d'Oinoanda, les rayons solaires viendront eux aussi animer la prière adressée au dieu hypsistos:

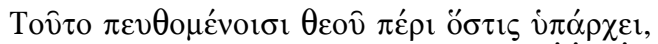

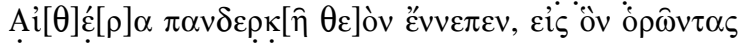

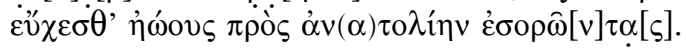

À ceux qui ont interrogé le dieu au sujet de ce qu'il est, il a répondu qu'Éther est le dieu qui voit tout, qu'il faut prier en le regardant, à l'aurore, en se tournant vers l'orient.

La disposition même de l'inscription la soumettait chaque jour au rayonnement du soleil levant ${ }^{52}$.

Autrement dit, on a là un point de passage entre les croyances et les pratiques gréco-romaines et égyptiennes, et il n'est pas anodin qu'un rituel du Serapeum d'Alexandrie, rapporté par Rufin d'Aquilée, ait intégré, aux côtés d'un mécanisme d'aimantation des statues de Sarapis et d'Hélios, un dispositif d'éclairage qui illuminait la statue cultuelle de Sarapis ${ }^{53}$.

Les représentations du dieu égypto-nubien Mandoulis, illuminées par les rayons du soleil, sont des signes du dieu, à la fois dans l'imaginaire gréco-romain et égyptien. Le rituel proprement égyptien pourra être perçu par le visiteur, à travers ses propres codes culturels, comme grec, et le dieu égyptien, rayonnant, sera accepté comme l'équivalent d'Hélios et d'Apollon ${ }^{54}$. Comme c'était le cas pour Thessalos, dans le

${ }^{51}$ Alliot 1954-1959, I, 413.

${ }^{52}$ Un autre oracle d'Apollon insiste sur le rôle des rayons solaires dans la révélation divine: voir Porphyre, cité par Eusèbe, Préparation évangélique V, 8, 11.

${ }_{53}$ Voir là-dessus Thélamon 1974; Rufin, Histoire Ecclésiastique II, 23.

${ }^{54}$ On sait, grâce à une figurine de terre cuite du musée du Louvre et à des représentations figurées sur des linceuls, qu'à l'époque impériale, l'enfant solaire a été iconographiquement traduit en grec par l'adjonction de rayons solaires et d'un nimbe à son image traditionnelle. Voir, pour la figurine: Paris, musée du Louvre, Inv. 
passage cité plus haut, l'effigie divine peut dès lors devenir le lieu de l'épiphanie du dieu, d'une véritable vision.

\section{Le signe comme manifestation}

Même si le texte est très corrompu, il est clair que Mandoulis se mon-

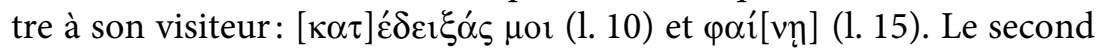
terme appartient au vocabulaire technique de l'apparition. Le verbe vev́( (1. 10) est lui aussi à mettre en relation avec la rencontre du dieu: il désigne un signe de la tête ou une inclinaison du corps, mais revêt dans le cadre oraculaire un sens technique que l'on rencontre dans les textes grecs comme égyptiens ${ }^{55}$. C'est bien toutefois la relation de vis-à-vis avec le dieu qui est soulignée ici, par un effet d'anthropomorphisme évident.

Avec le contenu de la vision, on quitte un instant l'ambiance "grecque» de la consultation. Ce passage pose de grosses difficultés de lecture, mais il est clair que se déploient plusieurs tableaux successifs, dont le premier est celui de la barque solaire parcourant le ciel $^{56}$.

Cette image évoque des traditions funéraires égyptiennes relatant le voyage nocturne du soleil dans l'au-delà ${ }^{57}$. C'est le motif central de l'ensemble des «Livres du monde inférieur» qui apparaissent au Nouvel Empire: durant la nuit, le soleil poursuit sa course en descendant sur sa barque dans une région inférieure du cosmos, qui est à la fois perçue comme l'au-delà, comme les profondeurs de la terre, mais aussi comme un monde obscur et aquatique, assimilé au Noun, l'«océan» primordial. Parcourant ce "Noun d'en bas», le soleil vieillissant va se régénérer, en même temps qu'il régénère les morts et leur roi, Osiris.

Cet univers apparait dans le Livre des Cavernes, dont des représentations ornent les parois de la tombe de Ramsès VI ${ }^{58}$. En effet, le

\footnotetext{
E $30249\left(\mathrm{II}^{\mathrm{e}}-\mathrm{III}^{\mathrm{e}}\right.$ siècle de notre ère, provenance inconnue); les linceuls, datés du $\mathrm{II}^{\mathrm{e}}$ siècle de notre ère proviennent de Saqqara: Le Caire, Musée égyptien, JE 59117; Cracovie, Musée archéologique, MAK/AS/2336. De nombreuses représentations d'Harpocrate radié sont connues par ailleurs.

55 Voir Dunand 2002. Les textes égyptiens parlent d' «approuver de la tête» ou de "dire non", semble-t-il en relation avec le mouvement de la barque divine en procession: soit le dieu approuve ou réprouve en avançant ou en reculant, soit il fait un choix, dans le cas de réponses multiples, en avançant vers l'ostracon où est inscrite la bonne réponse.

${ }^{56}$ Nous avons retenu ici la lecture de H. Lewy.

57 Hornung 1999, 26 et 83-95.

${ }^{58}$ Sur ce rapprochement: Lewy 1944.
} 
monde souterrain y est divisé en six secteurs successifs, six cavernes (qereret) d'où émerge le soleil au matin. Au cours de cette traversée, l'astre illumine le monde des morts, représentés dans leur sarcophage et qui pourraient être les $\delta \varepsilon \dot{\varepsilon} \mu(\mu) \alpha \tau \alpha$, les «formes» aperçues par notre visionnaire. Pendant la nuit terrestre, les défunts bienheureux jouissent de la lumière du soleil, dont sont définitivement privées les ombres des coupables. Le moment central est, dans le secteur 3, la rencontre de Rê avec Osiris en son sarcophage, qui initie la résurrection d'Osiris parallèlement à la régénération du soleil par Tatenen, dieu de la terre, dans le secteur 4. La navigation souterraine du soleil est aussi perçue comme un voyage dans la Nout d'en-bas, Nout étant la divinité du ciel, représentée dédoublée dans les illustrations du Livre du Jour et du Livre de la Nuit, au Nouvel Empire, par exemple dans la tombe de Ramsès VI. Il n'est donc pas incohérent, dans notre texte, de voir se déployer une vision à la fois souterraine et céleste du voyage nocturne

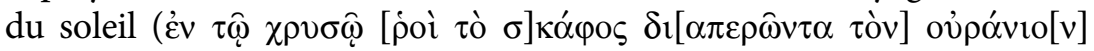
$\left.[\pi]{ }_{0} \lambda o v, 1.11-12\right)$. Dans la dernière section du livre funéraire égyptien sont représentées les deux collines dont émerge Rê: la barque solaire

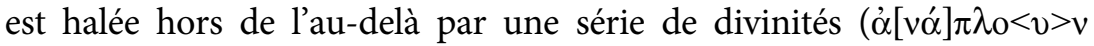

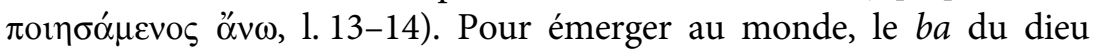

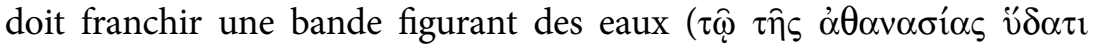

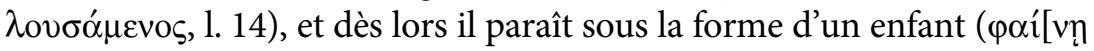
$\dot{\omega} \varsigma \pi \alpha 1 \delta i ́$ ov, 1. 15). Nous retrouvons bien là un certain nombre d'éléments présents dans notre texte, indépendamment même des restitutions de Hans Lewy, ce qui rend vraisemblable une initiation du visiteur aux traditions eschatologiques égyptiennes. En outre, l'image égyptienne du monde nocturne souterrain devait faire résonance, dans l'imaginaire d'une personne hellénisée, avec les cavernes de la nuit orphiques.

Au matin, donc, le soleil reparaît et, si l'on suit la restitution de Lewy, il reparaît sous la forme d'un enfant, conformément aux traditions égyptiennes. C'est alors, au petit jour, que le visiteur a pu porter son regard sur certaines scènes du temple et leur donner un sens enrichi par ses visions nocturnes.

De fait, cette image du soleil enfant émergeant de l'eau est très présente dans le temple de Kalabchah, où Mandoulis est représenté coiffé de la boucle de l'enfance, souvent le doigt à la bouche, accroupi ou assis, parfois sur une fleur et un bassin. Cette représentation renvoie à un mythe de création réputé originaire d'Hermopolis, qui associe la création du monde (la "Première Fois» en égyptien) au premier lever du soleil, sous la forme d'un enfant assis sur une fleur de lotus.

\section{(C) 2012 Koninklijke Brill NV}


La création du monde pour les Hermopolitains n'est donc que la première fois d'un cycle de levers du solei ${ }^{59}$. Cette image de l'enfant sur le lotus était complétée par des couleurs maintenant disparues, mais en partie enregistrées par Henri Gauthier: la peau de Mandoulis l'enfant était le plus souvent peinte en vert, couleur associée à la renaissance, ou parfois en noir, couleur du limon fertile du Nil et symbole de régénérescence. En outre, les représentations du dieu portent souvent les yeux incrustés: c'est par exemple le cas dans le pronaos, de chaque côté du mur menant au naos, et sur le mur ouest du déambulatoire. Elles évoquent là encore le mythe de l'enfant solaire et de son regard qui solidifie le monde et fait ainsi émerger la première butte de terre du magma primordial.

Le contenu même de la vision est donc proprement égyptien, et il y a loin de cette apparition de l'enfant solaire émergeant des cavernes de la nuit à l'identification de Mandoulis à l'Aiôn ou l'Apollon grecs. L'intervention d'intermédiaires était donc nécessaire. De fait, la présence des prêtres est partout perceptible: la disposition même des graffiti montre qu'un personnel du sanctuaire surveillait la préservation des espaces sacrés et tabous, soit en inscrivant lui-même les graffiti, soit en limitant les surfaces offertes aux inscriptions. En effet, aucun graffito n'a été retrouvé dans le naos et le pronaos, et tous sont concentrés dans un espace bien circonscrit. Bien plus, il est vraisemblable, selon nous, qu'outre les pratiques rituelles traditionnellement associées à la préparation à l'incubation, le visiteur avait reçu en préalable à la vision du dieu une sorte de «catéchèse» égyptienne dont le sens profond était activé par la vision proprement dite.

\section{Bilinguisme des signes}

À l'issue de cette cérémonie solaire et à partir de la vision du dieu, le visiteur affirme connaître l'identité de Mandoulis: "Alors je t'ai reconnu, Mandoulis, comme Hélios, le maître qui voit tout, le souverain universel, l'Aîon qui règne sur toutes choses ${ }^{60}$.»

\footnotetext{
${ }^{59}$ Sauneron 1959; Morenz \& Schubert 1954.

${ }^{60}$ Nous ne développerons pas ici la question des majuscules à mettre à $\ddot{\eta} \lambda$ ıov et $\alpha i \hat{\imath} v \alpha$ (s'agit-il d'Hélios et d'Aiôn, ou du soleil et de l'éternité?) qui pose des difficultés importantes mais est somme toute une question de modernes.
} 
La similarité d'un rituel comme celui du Serapeum d'Alexandrie a probablement joué un rôle fondamental dans le rapprochement entre Mandoulis et Hélios. Mais l'assimilation du dieu de Kalabchah avec l'Aiôn divin a peut-être elle aussi un fondement rituel.

En effet, une cérémonie annuelle, qui se déroulait durant la nuit du 5 au 6 janvier, date du solstice d'hiver, dans le Koreion d'Alexandrie, évoque très nettement celle de Kalabchah. Les détails en sont connus par un texte d'Épiphane de Salamine, datant de la deuxième moitié du $\mathrm{IV}^{\mathrm{e}}$ siècle de notre ère, qui relate qu'on y fêtait, durant toute la nuit, au son des chants et de la flûte, la naissance d'Aiôn ${ }^{61}$. Après le chant du coq, des porteurs de torches entraient dans une caverne souterraine (une chapelle souterraine du Koreion) et en remontaient sur un brancard une effigie en bois représentant un personnage masculin nu, assis, avec le sceau d'une croix sur le front, deux autres sur les mains, deux encore sur les genoux. On la transportait sept fois autour du centre du saint des saints, au rythme des flûtes, des tambourins et des chants, et on la remportait ensuite dans la caverne ${ }^{62}$.

Comme dans les textes de Kalabchah, il est ici question d'une "caverne souterraine» et d'un Goóvov, et le lever du jour revêt une importance particulière. Les rituels du temple de Mandoulis à Kalabchah ont pu être réinterprétés à la lumière de ces cérémonies alexandrines, dont Jean Yoyotte a démontré les origines égyptiennes. Une fois ce point de passage établi, la description du dieu en des termes propres à la religiosité en vogue alors dans l'Empire était facilitée.

Identifié à Hélios et Aiôn, Mandoulis est donc intégré à la vision gréco-romaine des dieux solaires: ses signes épiphaniques sont perçus comme bilingues et le dieu peut dès lors faire l'objet d'une traduction, d'une interpretatio Graeca.

Dès le début de l'hymne, l'adresse à Mandoulis comme "Titan» insiste sur sa parenté avec Hélios: si, chez Hésiode, Hélios n’est pas lui-même un Titan, il est le fils du Titan Hypérion et de la Titanide Theia. Mais surtout, les Hymnes Orphiques développent cette thématique. Ainsi, l'Hymne Orphique 8 s'adresse à Hélios en ces termes:

${ }^{61}$ Panarion LI, 22.

${ }^{62}$ Sur ce texte, voir Belayche 1984; Bowersock 1990, 22-28; Chuvin \& Yoyotte 2003, qui démontrent que le rituel du Koreion est beaucoup moins tardif que ne le pense G. Bowersock.

(C) 2012 Koninklijke Brill NV 


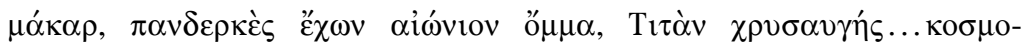

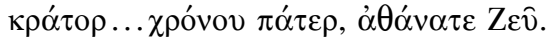

Bienheureux, qui voit tout avec son oil éternel, Titan à l'éclat de l'or, maître du monde, père du temps, Zeus immortel.

La parenté est extrêmement frappante entre ce texte et notre proscynème. On rencontre en effet dans notre hymne, de manière problé-

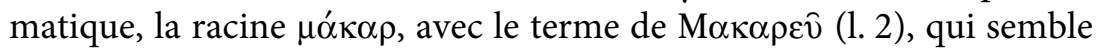
renvoyer à un nom propre, Makarée; il s'agirait d'un fils d'Hélios, également appelé Makar. Là où l'Hymne Orphique désigne Hélios comme

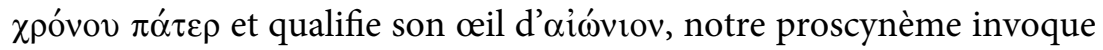

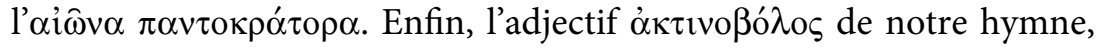
qui n'est pas attesté ailleurs à notre connaissance, développe l'image orphique du Titan $\chi \rho v \sigma \alpha v \gamma \eta \dot{s . ~}$

Le rapprochement opéré entre Aiôn, Apollon et Hélios dans les Papyrus Magiques grecs a par ailleurs contribué à l'assimilation de Mandoulis et d'Aiôn ${ }^{63}$, et ce sont les mêmes images que développe un hymne attribué à Mésomède, poète crétois de la cour d'Hadrien, où Hélios est explicitement rapproché d'Aiôn:

$\Sigma \grave{v} \delta$ '̉ $\lambda \alpha \mu \pi \rho \alpha \hat{\imath} \varsigma \dot{\alpha} \kappa \tau i ̂ \omega v$ $\gamma \alpha \hat{\imath} \alpha \nu \pi \hat{\alpha} \sigma \alpha v \pi v \rho \sigma \varepsilon v \dot{\omega} \omega v$ Aì̄v $\dot{\alpha} \sigma \beta \varepsilon \dot{\sigma} \sigma \tau \omega v \hat{\varphi} \lambda \sigma \gamma \mu \hat{\omega} v$

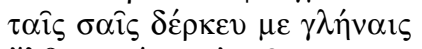

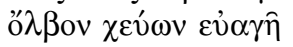
$\tau \widehat{\varphi} \sigma \hat{\varphi}, \Pi \alpha i o ́ v, \beta \alpha \kappa \chi \varepsilon v i \alpha$ ...Tiтóv

Toi qui avec des rayons éclatants illumines toute la terre, Aiôn aux flammes inextinguibles, regarde moi avec tes yeux, répandant une pure félicité sur ton Bacchant, Paian...Titan ${ }^{64}$.

La parenté des épithètes d'Hélios avec celles de l'adresse à Mandoulis de notre texte laisse penser que ce type d'images circulait largement dans l'Empire au $\mathrm{II}^{\mathrm{e}}$ et au $\mathrm{III}^{\mathrm{e}}$ siècle. Pour un individu de culture gréco-romaine qui voyait Mandoulis représenté comme un enfant sur une fleur de lotus, le rapprochement devenait possible avec l'Aiôn: on connaît ainsi une intaille magique, conservée à la Bibliothèque nationale de France, où l'enfant sur le lotus est désigné comme ó kúpros $\tau \hat{\eta} \varsigma$

\footnotetext{
63 Voir par exemple PGM, I, 262-247; IV, 1167-1226.

64 Texte donné dans Nock 1934, 96.
} 
oíкov $\mu \varepsilon ́ v \eta \varsigma^{65}$, titre qui apparaît comme celui de l'Aiôn Éternel dans un papyrus magique ${ }^{66}$.

Pour des commentateurs comme Arthur D. Nock, la rhétorique à l'œuvre dans les textes de Kalabchah pointe vers une production gréco-romaine de ces hymnes. Il faudrait chercher du côté d'hommes instruits et intéressés par la religion égyptienne, qui détenaient de hautes fonctions, tel par exemple, à l'époque d'Hadrien, un certain L. Julius Vestinus qui occupait la charge administrative de grand prêtre d'Alexandrie et de toute l'Égypte ${ }^{67}$. Les hauts gradés en inspection dans les garnisons pouvaient tout aussi bien jouer ce rôle. Ainsi, la théologie de l'Aiôn alexandrin convergeait très nettement avec un courant très fort à l'époque antonine dans la politique impériale: Aiôn apparaît en Égypte sous Antonin le Pieux (138-139) comme légende sur une monnaie représentant le phénix pour célébrer un nouveau cycle sothiaque; le phénix est nimbé et radié, avec dans le champ l'inscription AI $\Omega$ N. L'animal fabuleux, qui incarnait la durée illimitée et l'éternel retour, revêtait comme Aiôn une dimension solaire et l'usage politique du concept d'Aeternitas à l'époque antonine a pu faciliter ce rapprochement: les empereurs étant inuicti, ils furent parfois identifiés au soleil et qualifiés comme lui d'aeterni. Ces monnaies, qui font référence à une année de crue exceptionnelle, étaient censées véhiculer cette idéologie impériale, idéologie qui devait être particulièrement répandue dans les milieux militaires. La proximité de la garnison de Talmis pourrait alors expliquer ce développement tout alexandrin dans un temple nubien.

Cette vaste diffusion élargit d'autant le cercle des personnes susceptibles d'avoir produit les textes de Kalabchah. Il ne s'agit pas nécessairement de lettrés alexandrins. D'ailleurs, l'auteur de notre texte est tout aussi au fait des traditions égyptiennes du temple. Assimiler Mandoulis à Aiôn, c'est, comme le souligne Françoise Dunand, une façon d'exprimer une vision cyclique d'un «retour perpétuel, promesse de durabilité d'un monde qu'il recrée sans cesse ${ }^{68} »$ : c'est donner un habillage grec à des traditions tout à fait égyptiennes, à commencer par celle, déclinée à Kalabchah, de l'enfant solaire sur le lotus. À aucun moment, ni dans la vision cyclique du temps solaire

\footnotetext{
65 Delatte \& Derchain 1964, 119, nº 152.

66 PGM, XIII, 328.

${ }_{67}$ Cité par Nock 1934, 98.

${ }^{68}$ Dunand 2002, 30.
}

(C) 2012 Koninklijke Brill NV 
qui mène à rapprocher Mandoulis d'Aiôn, ni dans son assimilation à Apollon, présente dans de nombreux proscynèmes, on ne renonce complètement au fonds égyptien du dieu. Apollon, on le sait notamment par Hérodote et Élien, était considéré comme l'équivalent grec du dieu Horus. Or, souvenons-nous que l'hôte de Kalabchah est un dieu double. Les épithètes apolliniennes rappellent ici l'autre versant de la personnalité de Mandoulis, son aspect Horien. De surcroît, la vision d'un Apollon rayonnant s'accorde très bien avec le modèle du dieu Horus-Rê d'Edfou, qui a servi de modèle à l'élaboration de Mandoulis: en effet, à Edfou, ce dieu est souvent décrit comme un dieu qui lance ses rayons à la manière de flèches. Un équivalent égyptien

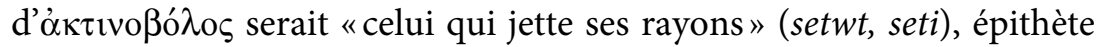
très fréquente d'Horus à Edfou.

Cette maîtrise des traditions égyptiennes nous semble le signe d'une production sacerdotale égyptienne, à une époque où le clergé égyptien pouvait chercher à assurer la promotion des cultes traditionnels en utilisant les nouveaux cadres de l'hellénisme ${ }^{69}$. Le récit de Thessalos faisait d'ailleurs état, à Thèbes et dans ses nombreux temples, de "prêtres amis des lettres et savants en maintes sciences». Un exemple particulièrement frappant de cet hellénisme sacerdotal nous est livré par les archives d'une famille attachée au temple de Min (Pan), à Panopolis, pendant la première moitié du $\mathrm{IV}^{\mathrm{e}}$ siècle de notre ère. Propriétaires de biens fonciers importants dans la région, ses membres portent souvent un nom double, romain et égyptien, et occupent des fonctions qui démontrent une aisance dans les deux sphères. Ainsi, le dénommé Horion, fils d'Aurelius Petearbeschinis et d'Aurelia Senpasis, prêtresse, est à la fois prêtre de Min et archiprophète de nome. Son frère Ammon est un érudit qui a laissé de sa main un fragment de l'Odyssée et une liste de philosophes grecs avec leurs disciples. Il se montre par ailleurs capable de se frayer un chemin dans la bureaucratie alexandrine quand il s'agit de négocier le statut sacerdotal de son neveu ${ }^{70}$. Un troisième frère, Harpocration, est panégyriste à la cour impériale ${ }^{71} \ldots$

${ }^{69}$ Nous renvoyons ici à la définition du concept d'hellénisme proposée par G. Bowersock: Bowersock 1990, 1-13. Voir Cameron 1965, 474-475, sur les prêtres poètes et sur la Thébaïde comme grand centre d'hellénisme à l'époque byzantine; Yoyotte 1969; Tait 1992; Clarysse 1993; Frankfurter 1998, 222-223; 2000; 2003.

${ }_{70}$ Willis 1978; 1979.

${ }^{71}$ Browne 1977. 


\section{A. Le bilinguisme des papyrus magiques}

Quant aux textes grecs avec lesquels des parallèles ont été proposés pour notre inscription, il apparait qu'hormis la proximité formelle avec certains oracles d'Asie Mineure, il s'agit majoritairement des soidisant « Papyrus Magiques Grecs», dont on sait qu'ils ont été composés pour beaucoup dans des milieux sacerdotaux égyptiens. La mythologie solaire égyptienne développée dans le cadre de notre oracle est ainsi reprise dans un papyrus magique où, pour obtenir un rêve oraculaire, on invoque «celui qui fait trembler, celui qui tonne, celui qui a avalé le serpent, qui accompagne la lune et heure par heure élève le disque solaire $^{72}$ ». En particulier, parmi ces Papyrus Magiques grecs, dix manuscrits en égyptien et en grec du $\mathrm{III}^{\mathrm{e}}$ siècle de notre ère, la collection Anastasi, viennent tous d'un même milieu sacerdotal thébain. L'association du démotique avec du vieux copte et du grec, souvent de la même main, dans plusieurs manuscrits implique l'existence de scribes et de lecteurs à l'aise en grec et en égyptien. Par ailleurs, la thématique des formules elles-mêmes reflète très nettement les traditions des temples et des maisons de vie ${ }^{73}$.

Ce travail de réinterprétation et d'adaptation des traditions et des textes anciens à des situations nouvelles, cette propension à intégrer un nouveau lexique, de nouveaux noms divins (Rê devient Hélios, Horus Apollon, etc.) et de nouvelles méthodes rituelles se situent dans le prolongement des activités traditionnelles des maisons de vie. Un des manuscrits de la collection Anastasi, qui combine des formules en grec, en démotique et en vieux copte, est d'ailleurs inscrit au dos d'un manuscrit démotique du «mythe de l'œil du Soleil ${ }^{74} »$. Ce texte mythologique, connu par des manuscrits démotiques, a fait l'objet d'une traduction grecque au $\mathrm{III}^{\mathrm{e}}$ siècle de notre ère, témoignant des capacités des scribes égyptiens à habiller leurs dieux à la grecque ${ }^{75}$.

Enfin, un aspect tout à fait fondamental de notre hymne, à savoir l'idée que la révélation divine plus que la rationalité permet d'accéder à la connaissance vraie et assurée, est présent dans le corpus des Papyrus Magiques Grecs ${ }^{76}$. Dans la magie, la révélation divine porte

72 PGM, VII, 366-368.

73 Tait 1995; Frankfurter 1998, 229.

${ }^{74}$ PGM, II = Leiden I 384v. Voir Ritner 1995, 3337-3338.

75 West 1969; de Cénival 1988.

${ }^{76}$ Ainsi, dans le Grand Papyrus magique de Paris, le dieu solaire apparaît sous la forme d'un jeune homme rayonnant et révèle les vérités du monde directement

\section{(C) 2012 Koninklijke Brill NV}


souvent sur le vrai nom de la divinité, car ce nom s'identifie à l'être même du dieu et sa connaissance permet au magicien de se rendre maître de toute la force divine - on rencontre cette thématique tout à fait égyptienne dans le mythe du «Nom caché de Rê», utilisé par la magicienne Isis pour prendre pouvoir sur lui. Dans notre document, le nom joue également un rôle central, dans la mesure où il est au fondement même de la démarche d'interpretatio.

Traduire en grec les traditions égyptiennes est devenu à l'époque gréco-romaine une ambition claire des prêtres égyptiens. Mais, comme l'a démontré David Frankfurter, le choix du fonds à traduire a sensiblement évolué depuis l'époque hellénistique où on reprenait surtout les anciens thèmes de la littérature royale, la Königsnovelle ${ }^{77}$. De plus en plus, les prêtres mettent l'accent sur la divination et la révélation directe du divin - un texte comme le Songe de Nectanébo montre bien l'articulation entre ces deux thématiques au début de l'époque impériale $^{78}$. Dans la collection Anastasi, on s'intéresse surtout aux charmes d'amour et aux rituels de révélation et de divination. "Le rituel sacerdotal archaïque de consultation de la volonté divine, le peh-netjer, devient une pratique presque mystique de rencontre directe du dieu par un rêve ou une vision ${ }^{79}$.»

\section{B. Les mutations de la pratique oraculaire}

Dans le cas qui nous occupe, Mandoulis est dès le départ un dieu oraculaire qui «vient à celui qui l'appelle», disent les textes hiéroglyphiques du temple, mais cet aspect fait ici l'objet d'un développement tout à fait intéressant. Les différents dieux gréco-romains dont il est rapproché sont des dieux oraculaires. Ainsi, Aiôn est une divinité liée à la maitrise du temps et du destin, comme l'exprime l'attribut du

au dédicant: $P G M, I V, 634-639$ et 710-715. On retrouve également cette thématique de la révélation comme source de la véritable connaissance dans un autre corpus de textes émanant des milieux sacerdotaux égyptiens, le Corpus Hermeticum, qui se donne souvent pour une révélation d'Hermès-Thoth apparaissant en songe à l'un de ses disciples.

77 Frankfurter 1998, 224-225.

${ }^{78}$ POxy. XI, 1381 ( $\mathrm{II}^{\mathrm{e}}$ siècle de notre ère). Tout en se maintenant dans la thématique de la Königsnovelle, ce récit insiste sur les réticences du héros à traduire en grec les traditions concernant Imhotep (Asclépios) et en particulier sur l'épiphanie du dieu, qui lui enjoint d'effectuer cette traduction. Il s'agit là d'une véritable propagande sacerdotale, dont on retrouve des éléments dans le dossier de Kalabchah: voir IM, 168, v. $24-25$ et $33-34$.

79 Frankfurter 1998, 230; voir sur cette question Ritner 1993, 214-220.

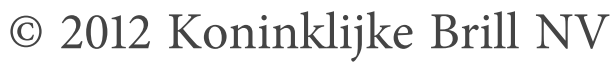


zodiaque qui lui est souvent prêté en tant que dieu chronokratôr et kosmokratô $r^{80}$. Assurant l'écoulement cyclique du temps, il est relié à la Tuchê et devient Heimarmenê, le Destin. C'est dans ce cadre que les textes magiques s'adressent à lui, insistant sur son aspect bienfaisant, providentiel, dans l'espoir de s'assurer sa bienveillance efficace ${ }^{81}$.

Par ailleurs, les épithètes que reçoit Mandoulis au début de l'hymne sont celles qui sont utilisées dans les autres proscynèmes en relation avec Apollon, qui n'est pas nommé ici: dans l'hymne de Maximus, Mandoulis est appelé "Phoibos Apollon», "Mandoulis Apollon Pythios» ailleurs. Le nom de "Titan", dans les hymnes orphiques ou magiques, désigne d'ailleurs souvent Apollon $^{82}$, et si l'adjectif $\mu \alpha ́ \alpha \alpha \rho$ qualifie diverses divinités, c'est particulièrement vrai d'Apollon, notamment dans l'épigramme de Maximus: l'invocation de Mandou-

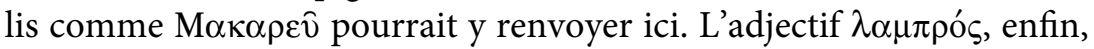
renvoie indirectement au dieu, dont une des épithètes récurrentes est $\lambda \alpha \mu \pi \rho{ }^{\prime} \tau \alpha \tau \sigma \varsigma^{83}$.

On cherche donc dans ces textes à promouvoir le visage oraculaire de Mandoulis. Comme l'a démontré David Frankfurter, ce qui jusque là était réservé à la classe sacerdotale dans le cadre d'une révélation privée s'étend à un public plus large, probablement du fait des très grandes difficultés que les temples égyptiens connaissent à partir du III $^{\mathrm{e}}$ siècle $^{84}$. Les prêtres de Kalabchah ont su saisir l'occasion de diffuser auprès de visiteurs curieux de nouvelles expériences religieuses, des connaissances et des pratiques jusque-là «ésotériques». On connaît plusieurs exemples de sanctuaires réaménagés pour permettre une consultation directe du dieu, comme c'est le cas à Abydos avec l'oracle de Bès ou encore vraisemblablement dans le temple d'Isis et Sarapis à Kysis, dans l'oasis de Kharga ${ }^{85}$. Il s'agissait de répondre à

${ }^{80}$ Voir, par exemple, une mosaïque du milieu du III $^{\mathrm{e}}$ siècle de notre ère à Antioche sur l'Oronte, dans ce qu'on a appelé la Maison des Aiôn: Levi 1947, I, 197-198 et II, pl. XLIIId; voir également Quet 1981.

81 Voir Belayche 1984, 18.

82 C'est le cas, par exemple, dans le PCorn. 21, 11, 269 (Philadelphie, an 25 de notre ère), où l'épithète est appliquée à Apollon Pythien.

${ }^{83}$ Ronchi 1974-1977, III, 642-643. Dans les autres textes de Kalabchah, il est explicitement attribué à Mandoulis-Apollon: IM, 168, 23. L'adjectif $\lambda \alpha \mu \pi \rho$ ó $\varsigma$ est également employé pour qualifier les joues de Mandoulis-Apollon: IM, 167, 11.

${ }^{84}$ Frankfurter 1998, 145-197; 2005; voir déjà Vernus 1977.

85 Après la fin du fonctionnement du culte, une chapelle a été ajoutée au sud du temple, dans la deuxième moitié du $\mathrm{IV}^{\mathrm{e}}$ siècle. Un espace a été aménagé, entouré d'un mur de briques enduit et décoré, et un trou fut ensuite percé dans le mur du temple

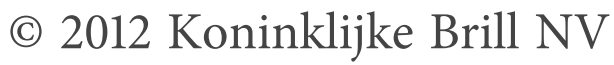


des attentes culturelles et religieuses nouvelles, et la présence d'une garnison militaire, à Talmis comme à Kysis, a probablement été l'élément déterminant de cette démarche des prêtres, avec laquelle elle est d'ailleurs chronologiquement concomitante.

Il faut donc revenir sur la nature et les objectifs des hymnes à Mandoulis. Que la vision de notre visiteur ou encore celle de Maximus aient réellement eu lieu importe peu, et il serait bien difficile de le dire. Ce qui nous semble plus porteur de sens, c'est que ces textes ont été rédigés de la même main, dans un cadre contrôlé par le clergé. Il semble même hautement probable que les prêtres en soient les auteurs ${ }^{86}$. L'usage du grec semble parallèlement indiquer une volonté de diffusion la plus étendue possible. Comme dans tout proscynème, la lecture du texte contribue à en réactiver les effets, mais ne s'agit-il pas aussi pour les prêtres d'assurer une certaine publicité à leur consultation oraculaire auprès d'éventuels visiteurs? Ne faudrait-il pas distinguer les proscynèmes courts et classiques, vraisemblablement laissés par des soldats à la suite de leur passage dans le temple, de ces proscynèmes longs et développés? De fait, un aspect surprenant de ces textes, pour lesquels nous avons souligné les grandes similitudes avec certains documents du Corpus Hermeticum et des Papyrus Magiques, est, d'une part, qu'ils sont inscrits dans un temple et ne relèvent pas de la sphère exclusivement privée, et d'autre part, qu'ils rendent publique une rencontre dont la teneur est en général maintenue secrète - même si l'objet de la révélation, de la compréhension même est ici éludé. En effet, dans les autres textes connus, le fidèle élu pour la révélation reçoit la recommandation de garder le silence sur les doctrines révélées: André Jean Festugière souligne l'existence d'une véritable loi du silence dans l'hermétisme ${ }^{87}$, mais aussi dans les Papyrus Magiques ${ }^{88}$, ou encore dans la révélation du médecin Thessalos. C'est loin d'être le cas ici, et la présence du texte dans la cour du temple nous incite à penser qu'on a affaire à un véritable appareil de promotion dans lequel pourraient prendre place les «conseils de piété» de Sansnos. On peut

entre la chapelle de briques et le sanctuaire intérieur, offrant un dispositif pour un oracle parlant en direction de la nouvelle chapelle: Dunand 1991, 246; Frankfurter 1998, 167-168; Frankfurter 2005.

${ }^{86}$ F. Dunand a d'ailleurs souligné l'importance, à cet égard, de la célébration de la «sainte Talmis» et de la divinité principale de la région, Isis, à la fin du proscynème: Dunand 2002, 38, n. 68.

${ }_{87}$ Festugière 1939, 50.

${ }^{88}$ Par exemple PGM, IV, 556-560.

(C) 2012 Koninklijke Brill NV 
d'ailleurs se demander si ce dernier ne faisait pas partie des membres du clergé disponibles dans le temple pour encadrer la consultation. Il fallait bien en effet des interprètes des signes du dieu, interprètes dont la présence est gommée dans le texte pour restituer l'immédiateté de la rencontre avec Mandoulis.

Un autre élément important nous semble être une fresque dont des restes situés dans la cour à deux pas de notre proscynème, sur l'entrecolonnement de la façade de la salle hypostyle, ont été photographiés par Henri Gauthier. On entrevoit une représentation d'un personnage anthropomorphe à tête de faucon, vêtu en soldat et nimbé. Henri Gauthier précise que cette peinture ne peut être datée de l'époque copte car elle se trouvait sous l'enduit que les chrétiens ont appliqué sur les murs de Kalabchah avant d'y peindre leurs propres représentations. Cette divinité nimbée évoque des figurations d'Horus soldat nimbées et radiées, dont l'une en particulier semble liée à un culte oraculaire : c'est la seule statue "parlante» connue en Égypte et elle s'intégrait vraisemblablement à un oracle parlant, fonctionnant tout particulièrement dans un contexte militaire (fig. 6$)^{89}$. Pour un public de soldats, ce type de représentation pouvait être éloquent sur la nature du dieu du sanctuaire et contribuer à éveiller une pieuse curiosité.

Les conseils de piété de Sansnos, appelant les visiteurs à honorer les dieux locaux, et cette peinture de faucon militaire nimbé fonctionnaient, selon nous, en complément de la description de Mandoulis dans notre hymne: la fresque renvoie bien à un Mandoulis rayonnant et apollinien, sous la forme d'Horus, l'Apollon égyptien. Une telle peinture n'a pu être réalisée qu'avec l'accord, et même probablement sur la commande des prêtres: ce faisant, ils ont reconstruit un nouveau

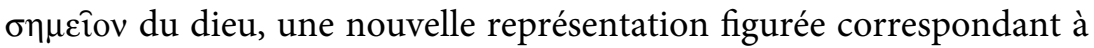
son nouveau public ${ }^{90}$.

Tous ces documents témoignent de l'ampleur de l'innovation religieuse dont ont su faire preuve les clergés égyptiens à l'époque impériale. Il n'est pas anodin qu'ils aient concentré leur intérêt sur la consultation oraculaire. La divination traditionnelle dans le cadre des

89 Loukianoff 1936.

90 Un autre cas de transformation de l'iconographie des dieux dans le cadre du temple est visible dans le mammisi du dieu Toutou à Ismant el-Kharab (Kellis), actuellement fouillé par O. Kaper dans le cadre du Dakhlah Oasis Project. Par ailleurs, les hymnes d'Isidoros dans le temple d'Isis-Renenoutet à Narmouthis, dans le Fayoum, mettent également en valeur la démarche de traduction pour les Grecs des traditions égyptiennes, sans doute destinée à attirer un public nouveau. 


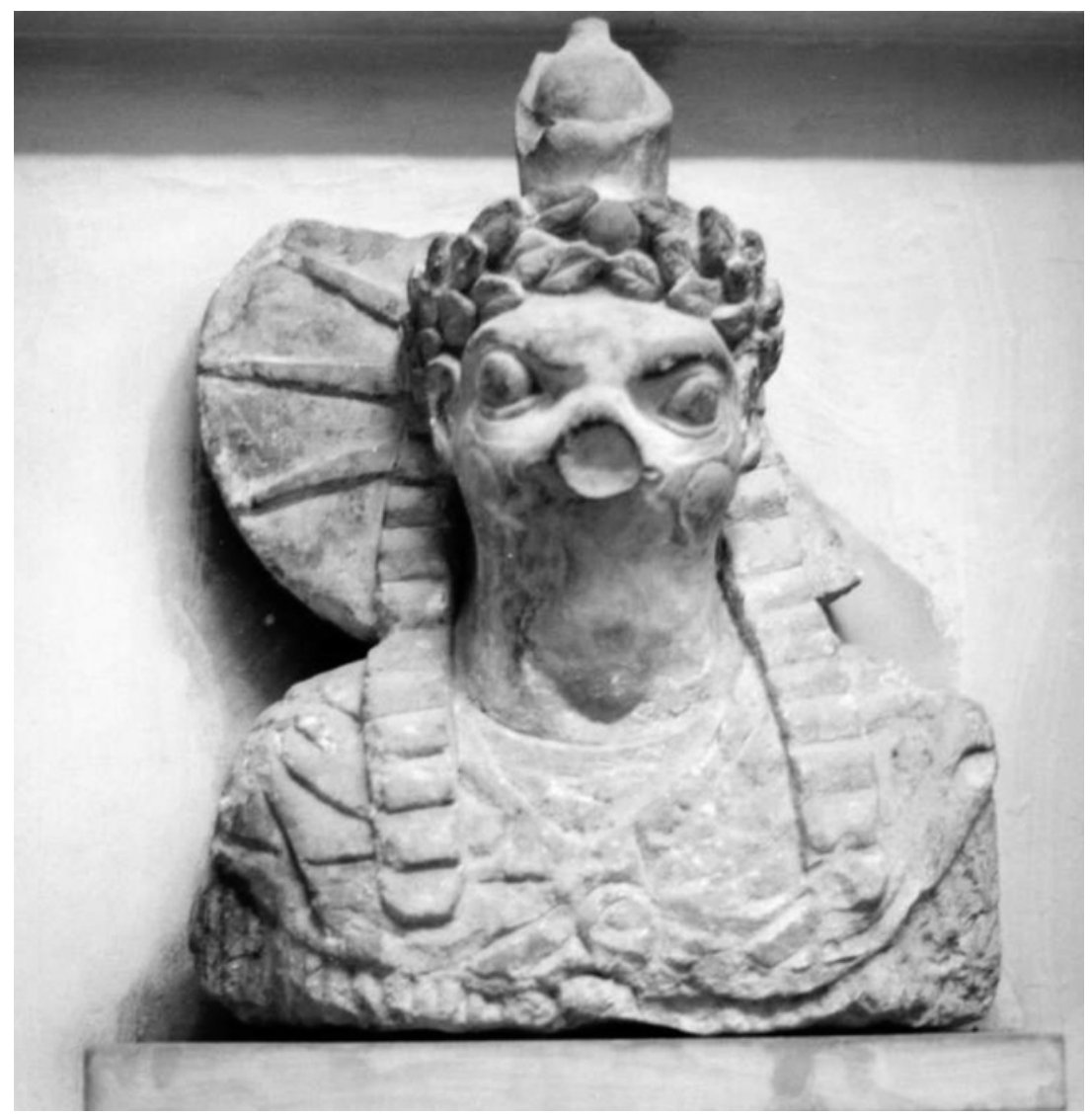

Fig. 6 Une statue parlante. Photographie G. Tallet, avec l'aimable autorisation du Musée égyptien du Caire.

processions de la barque divine, trop exceptionnelle pour répondre aux attentes permanentes des fidèles et probablement trop coûteuse en une période de fort déclin économique des temples, cède ici la place, sans nécessairement disparaître pour autant, à une nouvelle technique rituelle. L'évolution de la relation du fidèle au dieu dans le sens d'une piété personnelle, amorcée en Égypte depuis le Nouvel Empire ${ }^{91}$, s'adapte à une curiosité que des individus comme Jamblique ont par la suite emblématisée ${ }^{92}$. La concurrence était par ailleurs forte avec

\footnotetext{
91 Frankfurter 1998, 145-197.

92 Carlier 1974; Athanassiadi 1993.
}

\section{(C) 2012 Koninklijke Brill NV}


d'autres modèles religieux alors en plein essor, et il importait d'assurer la promotion de son propre dieu.

Tout en ouvrant le temple sur l'extérieur, les prêtres ont toujours su en contrôler les espaces interdits. Ils n'ont pas abandonné les croyances traditionnelles en les adaptant à la nouvelle lingua franca, le grec ${ }^{93}$, mais ont par là même démocratisé l'accès aux mystères divins et rendu la parole du dieu lisible par tous, au point d'en faire un medium de promotion de leur consultation oraculaire. Pour autant, les lettres inscrites sur les parois du temple ne perdent pas leur valeur numineuse. Les hiéroglyphes demeurent les medou-netjer, les paroles des dieux: ils sont l'écriture du monde ${ }^{94}$. Et même si le grec remplace peu à peu l'égyptien, même dans les milieux sacerdotaux les plus conservateurs, une véritable fascination, voire une vénération pour les signes hiéroglyphiques perdure. Ainsi, dans la famille très hellénisée d'Horapollon, au IV ${ }^{\mathrm{e}}$ siècle de notre ère, alors qu'on enterre son oncle Heraiskos «selon les coutumes appropriées pour les prêtres», miraculeusement, des hiéroglyphes lumineux apparaissent sur le linceul ${ }^{95}$. Le caractère presque magique de ces signes, que l'on constate d'ailleurs sur de nombreux linceuls de la même époque (certains sont ornés de pseudohiéroglyphes), va précisément de pair avec une compréhension fantaisiste de leur signification, dont l'ouvrage d'Horapollon donne un bon exemple.

Ce sont peut-être ces $\sigma \eta \mu(\varepsilon) \hat{\imath} \alpha \lambda \alpha \mu \pi \rho \alpha$, lumineux, porteurs d'un éclat divin mais qu'on comprend de moins en moins, qu'évoque le début de notre hymne. On en verra une récupération magique dans les charaktêres des gemmes gravées ${ }^{96}$.

En définitive, l'obtention des signes de Mandoulis a contribué à établir un lien entre deux mondes qui coexistent de fait sur les murs de Kalabchah mais ne se rencontrent pas: ils s'expriment d'une part à travers les textes hiéroglyphiques du temple, d'autre part à travers les graffiti grecs des visiteurs, et investissent des espaces différents. Les textes hiéroglyphiques se développent en particulier dans le naos et le pronaos, espaces interdits d'accès aux visiteurs, tandis que les graffiti

\footnotetext{
93 Voir Fowden 1986, 43-44; Bowersock 1990, 1-13.

94 Voir, sur cette question, Vernant (dir.) 1974 et la contribution de J.-J. Glassner dans le présent volume.

${ }_{95}$ Damascius, fr. 174.

96 Voir, dans le présent volume, la contribution d'A. Mastrocinque.
} 
grecs s'installent dans des espaces plus ouverts, comme le mur d'enceinte, la cour ou le déambulatoire à l'extérieur du naos, dont le décor est pour l'essentiel inachevé et où les textes hiéroglyphiques n'ont pas été gravés. D’une certaine manière, en faisant inscrire ces hymnes, les prêtres égyptiens auront continué l'entreprise de décor du sanctuaire, demeurée inachevée faute de soutien impérial. Mais ils l'auront fait dans la nouvelle langue et selon les nouveaux codes de la société dans laquelle ils vivaient. Les graffiti nubiens qui ornent les murs du temple et témoignent de la perpétuation de l'oracle de Kalabchah après la fermeture du temple, jusqu'au $\mathrm{v}^{\mathrm{e}}$ siècle de notre ère, attestent la réussite de leur entreprise.

\section{Bibliographie}

Accorinti, D. \& Chuvin, P. (dir.) 2003, Des Géants à Dionysos. Mélanges offerts à F. Vian, Alexandrie: Edizioni dell'Orso.

Alliot, M. 1954-1959, Le culte d'Horus à Edfou au temps des Ptolémées, Le Caire: Institut Français d'Archéologie Orientale (Bibliothèque d'études de l'IFAO 20).

Arnold, D. 1975, Die Tempel von Kalabsha, Wiesbaden-Berlin: Deutsches Archäologisches Institut, Abteilung Kairo.

Assmann, J. 1978, «Eine Traumoffenbarung der Göttin Hathor: Zeugnisse "Persönlicher Frömmigkeit" in thebanischen Privatgräbern der Ramessidenzeit», Revue d'égyptologie 30, 22-50.

Athanassiadi, P. 1993, "Dreams, Theurgy and Freelance Divination: The Testimony of Iamblichus», Journal of Roman Studies 83, 115-130.

Beatrice, P. F. 2001, Anonymi Monophysitae Theosophia. An Attempt at Reconstruction, Leyde: Brill (Supplements to Vigiliae Christianae 56).

Belayche, N. 1984, "Aiôn: vers une sublimation du temps", dans Le temps chrétien de la fin de l'Antiquité au Moyen Âge, II ${ }^{e}-\mathrm{XIII}^{e}$ siècles, Paris 9-12 mars 1981, Paris: CNRS, 11-29.

Bernand, A. 1988, «Le statut de l'image divine dans l'Égypte hellénistique», dans Mélanges Pierre Lévêque, I, Besançon: Université de Besançon/Les Belles Lettres, 33-47.

Blackman, A. M. \& Fairman, H. 1942, "The Myth of Horus at Edfu. II», Journal of Egyptian Archaeology 28, 1942, 75-91.

Boespflug, F. \& Dunand, F. (dir.) 1997, Le comparatisme en histoire des religions, Paris: Cerf.

—. 2002, Voir les dieux, voir Dieu, Strasbourg: Presses Universitaires de Strasbourg.

Bowersock, G. W. 1990, Hellenism in Late Antiquity, Ann Arbor: The University of Michigan Press.

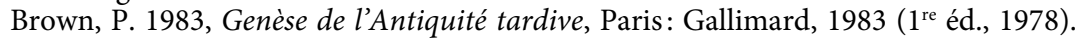

Browne, G. M. 1977, "Harpocration Panegyrista», Illinois Classical Studies 2, 184196.

Burstein, S. M. 1998, «Paccius Maximus: A Greek Poet in Nubia or a Nubian Greek Poet?», Cahiers de recherches de l'Institut de papyrologie et d'égyptologie de Lille $17 / 3,47-52$.

Busine, A. 2005, Paroles d'Apollon. Pratiques et traditions oraculaires dans l'Antiquité tardive (II ${ }^{e}-V I^{e}$ siècles), Leyde-Boston: Brill. 
Cameron, A. 1965, "Wandering Poets: A Literary Movement in Byzantine Egypt», Historia 14, 470-509.

Carlier, J. 1974, «Science divine et raison humaine», dans Vernant (dir.) 1974, 249263.

Caseau, B., Cheynet, J.-Cl. \& Déroche, V. (dir.) 2006, Pèlerinages et lieux saints dans l'Antiquité et le Moyen Âge. Mélanges offerts à P. Maraval, Paris: Centre de recherche d'histoire et civilisation de Byzance.

Cénival, F. de 1988, Le mythe de l'œil du soleil, Sommerhausen: Zauzich (Demotische Studien 9).

Chantraine, P. 1968, Dictionnaire étymologique de la langue grecque, Paris: Klinksieck.

Chuvin, P. \& Yoyotte, J. 2003, "Autour du solstice d'hiver: Épiphane et les fêtes alexandrines de l'Éternité», dans Accorinti \& Chuvin (dir.) 2003, 135-145.

Clarysse, W. 1992, "Some Greeks in Egypt», dans Johnson (dir.) 1992, 51-56.

- 1993, «Egyptian Scribes Writing Greek», Chronique d'Égypte 69, 186-201.

Curto, S., Maragioglio, V. \& Rinaldi, C. A. 1965, Kalabsha, Rome (Orientis Antiqui Coll. V).

Daumas, F. 1970, La Ouabet de Kalabcha, Le Caire: Centre de documentation et d'études de l'ancienne Égypte.

Delatte, A., \& Derchain, P. 1964, Bibliothèque nationale, Cabinet des médailles et antiques. Les intailles magiques gréco-égyptiennes, Paris: Bibliothèque nationale de France.

Derchain, P. 1965, Le Papyrus Salt 825 (BM 10051), rituel pour la conservation de la vie en Égypte, Bruxelles: Palais des académies (Mémoires de l'Académie royale de Belgique 58).

Desroches-Noblecourt, C. 1985, «Les zélateurs de Mandoulis et les maîtres de Ballana et de Qustul», dans Mélanges Gamal Eddin Mokhtar, I, Le Caire: Institut Français d'Archéologie Orientale (Bibliothèque d'études de l'IFAO 97), 199-218.

Dunand, F. 1991, "Miracles et guérisons en Égypte tardive», dans Fick \& Carrière (dir.) 1991, 235-250.

—. 1997, "Lieu sacré païen et lieu sacré chrétien. Autour des pèlerinages», dans Boespflug \& Dunand (dir.) 1997, 239-258.

—. 2002, "Le désir de connaître Dieu. Une vision de Mandoulis au temple de Kalabscha», dans Boespflug \& Dunand (dir.) 2002, 23-38.

Dunand, F., Wirth, J. \& Spieser, J.-M. (dir.) 1991, L'Image et la production du Sacré, Paris: Méridiens/Klincksieck.

Edfou I: De Rochemonteix, M., Chassinat, É., Le temple d'Edfou, I, Le Caire: Institut Français d'Archéologie Orientale, 1987 ( $2^{\mathrm{e}}$ éd. revue et corrigée par S. Cauville et D. Devauchelle).

Edfou IV: Chassinat, É., Le temple d'Edfou, IV, Le Caire: Institut Français d'Archéologie Orientale, 1929.

Elsner, J. \& Rutherford, I. (dir.) 2006, Pilgrimage in Graeco-Roman and Early Christian Antiquity. Seeing the Gods, Oxford: Oxford University Press.

Festugière, A. J. 1939, "L'expérience religieuse du médecin Thessalos", Revue Biblique $48,45-77$.

- 1950, La révélation d'Hermès Trismégiste, I, Paris: Les Belles Lettres.

FHN: Eide, T., Hägg, T., Pierce, R. H. \& Török, L. (éd.), Fontes Historiae Nubiorum. Textual Sources for the History of the Middle Nile Region between the Eighth Century $B C$ and the Sixth Century AD, 4 vol., Bergen: University of Bergen, 1994-2000.

Fick, N. \& Carrière, J.-C. (dir.) 1991, Mélanges Étienne Bernand, Paris: Les Belles Lettres.

Fowden, G. 1986, The Egyptian Hermes. An Historical Approach to the Late Pagan Mind, Cambridge: Cambridge University Press.

Frankfurter, D. 1998, Religion in Roman Egypt: Assimilation and Resistance, Princeton: Princeton University Press.

\section{(C) 2012 Koninklijke Brill NV}


(dir.) 1998, Pilgrimage and Holy Space in Late Antique Egypt, Leyde: Brill.

2000, «The Consequences of Hellenism in Late Antique Egypt: Religious Worlds and Actors", Archiv für Religionsgeschichte 2/2, 162-194.

- 2003, "Syncretism and the Holy Man in Late Antique Egypt», Journal of Early Christian Studies 11/3, 339-385.

- 2005, "Voices, Books and Dreams: The Diversification of Divination Media in Late Antique Egypt», dans Johnston \& Struck (dir.) 2005, 233-254.

- $2006 \mathrm{a}$, «Espaces et pèlerinages dans l'Égypte de l'Antiquité tardive», dans Caseau, Cheynet \& Déroche (dir.) 2006, 203-221.

- 2006 b, "Urban Shrines and Rural Saint in Fifth-Century Alexandria», dans Elsner \& Rutherford (dir.) 2006, 436-449.

Gauthier, H. 1909, "Cinq inscriptions grecques de Kalabchah (Nubie)», Annales du Service des Antiquités d'Égypte 10, 68-76.

—. 1911, Le temple de Kalabchah, Le Caire: Institut Français d'Archéologie Orientale.

Griffiths, F. L. 1929, "Meroitic Studies VI», Journal of Egyptian Archaeology 15, 69-74.

Habachi, L. 1969, "Divinities Adored in the Area of Kalabsha, with a Special Reference to the Goddess Miket», Mitteilungen des Deutschen archäologischen Instituts, Abteilung Kairo 24, 169-183.

Hölbl, G. 2004, Altägypten im Römischen Reich. Der römische Pharao und seine Tempel, II. Die Tempel des römischen Nubien, Mayence: von Zabern.

Hope, C. et alii 1989, «Dakhleh Oasis Project: Ismant el-Kharab 1991-1992», Journal of the Society for the Study of Egyptian Antiquities 19, 1-26.

Hornung, E. 1996, L'esprit du temps des pharaons, Paris, 1996: Lebaud ( $1^{\text {re }}$ éd., 1989).

- 1999, The Ancient Egyptian Books of the Afterlife, Ithaca-Londres: Cornell University Press.

IM: Bernand, É., Inscriptions métriques de l'Égypte gréco-romaine, Paris: Les Belles Lettres, 1969.

Johnson, J. H. (dir.) 1992, Life in a Multi-Cultural Society. Egypt from Cambyses to Constantine and Beyond, Chicago: The University of Chicago Press.

Johnston, S. I. \& Struck, P. T. (dir.) 2005, Mantikê. Studies in Ancient Divination, Leyde-Boston: Brill.

Kakosy, L. 1964, «Osiris-Aiôn», Oriens Antiquus 3, 15-25.

- 1978, «Einige Probleme des Ägyptischen Zeitbegriffes», Oikumene 2, 95-111.

Levi, D. 1947, Antioch Mosaic Pavements, 2 vol., Princeton: Princeton University Press.

Lewy, H. 1944, "A Dream of Mandulis», Annales du Service des Antiquités d'Égypte $44,227-234$.

Liddell, H. J., Scott, R., Jones, H. S. 1996, Greek-English Lexicon, Oxford: Clarendon Press.

Loukianoff, G. 1936, «Une statue parlante ou oracle du dieu Ré-Harmakhis», Annales du Service des Antiquités d'Égypte 36, 187-193.

Merkelbach, R. \& Stauber, J. 1996, «Die Orakel des Apollon von Klaros», Epigraphica Anatolica 27, 1-53.

Morenz, S. \& Schubert, J. 1954, Der Gott auf der Blume, eine Ägyptische Kosmogonie und ihre weltweite Bildwirkung, Ascona: Artibus Asiae.

Nock, A. D. 1928, "Oracles théologiques», Revue des études anciennes 30, 280-290.

- 1934, "A Vision of Mandulis Aion», Harvard Theological Review 27, 53-104.

OGIS: Dittenberger, W., Orientis Graecae Inscriptiones Selectae, Leipzig: Hirzel, 19031905.

PGM: Preisendanz, K. (éd.), Papyri Graecae Magicae. Die griechischen Zauberpapyri, Leipzig-Berlin: Teubner, 1928-1931 ( $2^{\mathrm{e}}$ éd. par A. Henrichs, Stuttgart, 1973).

\section{(C) 2012 Koninklijke Brill NV}


Preisigke, Wb: Preisigke, F., Wörterbuch der Grieschichen Papyrusurkunden, Heidelberg-Berlin: G. Preisigke, 1924-1931.

Puchstein, O. 1880, Epigrammata Graeca in Aegypto, Strasbourg: Truebner.

Quet, M.-H. 1981, La mosaïque cosmologique de Mérida: propositions de lecture, Paris: De Boccard (Publications du Centre Pierre Paris 6).

Quirke, S. (dir.) 1997, The Temple in Ancient Egypt. New Discoveries and Recent Researches, Londres: British Museum Press.

Ritner, R. K. 1993, The Mechanics of Ancient Egyptian Magical Practice, Chicago: Oriental Institute of University of Chicago.

—. 1995, «Egyptian Magical Practice under the Roman Empire: The Demotic Spells and Their Religious Context», dans Aufstieg und Niedergang der römischen Welt, II, 18, 5, Berlin-New York, 3333-3379.

Robert, L. 1971, «Un oracle gravé à Oinoanda», Comptes rendus de l'Académie des Inscriptions et Belles-Lettres, 597-619.

Ronchi, G. 1974-1977, Lexicon Theonymon rerum sacrarum et divinarum ad Aegyptum pertinentium quae in papyris ostracis titulis Graecis Latinisque in Aegypto repertis laudantur, 5 vol., Milan: La Goliardica.

Rutherford, I. 1998, "Island of the Extremity: Space, Language and Power in the Pilgrimage Traditions of Philae», dans Frankfurter (dir.) 1998, 229-256.

Sauneron, S. 1959, «Les Songes et leur interprétation dans l'Égypte ancienne», dans Les Songes et leur interprétation, Paris: Seuil, 17-61.

Säve-Söderbergh, T., 1953, On Egyptian Representation of Hippopotamus Hunting as Religious Motive, Upsala: C. W. K. Gleerup (Horae Soederblomianae 3).

SB: Preisigke, F., Sammelbuch griechischer Urkunden aus Ägypten, Wiesbaden: Harrassowitz, 1915-.

Stock, H. \& Siegler, K. G. 1965, Kalabsha, der grösste Tempel Nubiens und das Abenteuer seiner Rettung, Wiesbaden: Brockhaus.

Tait, W. J. 1992, "Demotic Literature and Egyptian Society", dans Johnson (dir.) 1992, 303-310.

- 1995, «Theban Magic», dans Vleeming (dir.) 1995, 169-182.

Thélamon, F. 1974, "Sérapis et le baiser du soleil», Antichità alto-adriatiche (Aquileia e l'Africa), 5, 227-250.

Traunecker, C. 1979, «Manifestations de piété personnelle à Karnak», Bulletin de la Société française d'égyptologie 85, 1979, 22-31.

_. 1991, "Observations sur le décor des temples égyptiens», dans Dunand, Wirth \& Spieser (dir.) 1991, 77-101.

—. 1997, «Lessons from the Upper Egyptian Temple of el-Qal'a», dans Quirke (dir.) 1997, 168-178.

Van den Plas, D. 1989, " "Voir” Dieu. Quelques observations au sujet de la fonction des sens dans le culte et la dévotion de l'Égypte ancienne», Bulletin de la Société française d'égyptologie 115, 4-35.

Vernant, J.-P. (dir.) 1974, Divination et rationalité, Paris: Seuil.

Vernus, P. 1977, «Le dieu personnel dans l'Égypte pharaonique», dans Centre interdisciplinaire d'étude de l'évolution des idées, des sciences et des techniques. Colloques de la Société Ernest-Renan, Orsay, 143-157.

Vleeming, S. P. (dir.) 1995, Hundred-Gated Thebes. Acts of a Colloquium on Thebes and the Theban Area in the graeco-Roman Period (P. Lugd.-Bat. 27), Leyde: Brill (Papyrologica Lugduno-Batava 27).

Volokhine, Y. 1998, "Les déplacements pieux en Égypte pharaonique: sites et pratiques cultuelles", dans Frankfurter (dir.) 1998, 51-97.

West, S. 1969, "The Greek Version of the Legend of Tefnut», Journal of Egyptian Archaeology 55, 161-183.

Willis, W. H. 1978, "Two Literary Papyri in an Archive from Panopolis», Illinois Classical Studies 3, 140-153.

\section{(C) 2012 Koninklijke Brill NV}


- 1979, "The Letter of Ammon of Panopolis to his Mother», dans Actes du $X V^{e}$ Congrès international de papyrologie, Bruxelles (Papyrologia Bruxellensa 17), 98-115.

Yoyotte, J. 1960, «Les pèlerinages dans l'Égypte ancienne», dans Les pèlerinages, Paris: Seuil (Sources Orientales 3), 17-74.

_. 1969, «Bakhtis: religion égyptienne et culture grecque à Edfou», dans Religions en Égypte hellénistique et romaine (Colloque de Strasbourg, 16-18 mai 1967), Paris: Presses Universitaires de France, 127-141. 